\title{
Abstracts of the 8th Congress of ESACP, the 15th International Congress of ISDQP and the 6th Congress of the European Group of Telepathology, Heraclion, Crete, 14-21 September 2002
}

\section{Oral presentations}

1

\author{
TELEPATHOLOGY - APPLICATION \\ SCENARIOS AND THE APPROPRIATE \\ EQUIPMENT \\ Schwarzmann P., Lopez L. \\ University of Stuttgart, Germany
}

A sufficient amount of experiences gained in telepathology until today allows to review application scenarios and equipment. Also many applications have reached daily routine, many scenarios are still the object of extended fieldtests, experimental investigations and of controversial discussions.

The spectrum of services is widespread and includes different applications:

- Fully remote diagnosis facilities e.g., intraoperational frozen section diagnosis.

- Second opinion scenarios.

- Expert teleconferences for primary and continuing education.

- Scientific applications: studies with extended participating groups.

- Exploitation of centralized services to improve diagnostic procedures.

To meet the needs of users, manufacturers have developed dedicated equipment according to the requests of the community of pathologists. On the other hand, existing freeware tools have stimulated pathologists to design their own facilities.

In the commercial sector today we find in principle two types of systems:
- Upper end systems for online telediagnosis with robotic, remotely operated microscopes, macrofacilities to assist in specimen preparation, archiving facilities and interfaces to HIS.

- Still image or passive systems preferentially for 2nd opinion applications, where images are collected and then send to the partner, who normally inspects the images offline and returns his opinion.

Still under controversial discussion are the minimum requirements for the different telepathology services. This is with respect to image quality (spatial resolution, spectral resolution, field of view), adequate data security and confidentiality requirements and the minimum requirements to the telecommunication link (online facility, bandwidth, costs, availability of networks).

New upcoming efforts to improve the efficiency of telepathology are being made to reach an optimal integration in the clinical workflow (integration in, or interfaces to the hospital information system) and finally the interoperability of equipment of different manufacturers and of different types.

\section{2}

\section{RECENT PROGRESS IN TELEMEDICINE ACTIVITIES IN RUSSIA}

Buravkov $\mathrm{S}$.

Faculty of fundamental medicine, Moscow State University, Russia

During last several years great increase of telemedicine activities could be noted in different regions of Russian 
Federation. Growing of telecommunication infrastructure, especially Internet technologies and access even in remote areas of Russia causes this interest of medical persons to modern technologies in medicine. More physicians utilize networks to solve their information problems finding the newest and the most important electronic versions of books and journals in everyday work. Four years ago we could find only 4 sites of telemedicine activity in former Soviet Union countries. Now their number increases drastically and reaches more than 30 sites, where telemedical technologies apply on regular basis.

Analyzing these activities we may say that most of them utilized videoconferences to perform teleconsultations. At the same time several sites based on delayed basis using WWW-servers, where medical information posted by qualified physician in order to improve diagnosis and future treatment. Multimedia techniques more often used in tele-education, providing approximately the same quality of education as in leading high school institutions (Moscow State University), expanding student auditorium. On other side the audiovisual contacts between teacher and student are necessary for education to be successful.

Although telemedicine meets a lot of problems associated with financial, subjective and other reasons it becomes more and more available in countries of former Soviet Union. At present time the most important point is the coordination and cooperation of these efforts in order to unify the protocols and scenarios of telemedicine consultations. This is of special concern for international telemedicine work taking into account the differences of medical approaches based on historical traditions and rules. We conclude that telemedicine could be the matter of choice when qualified physician and patient are separated by long distance and there is no other possibility to provide with high-qualified medical care.

\section{3}

COMPARISON OF TWO TELE-PATHOLOGY SYSTEMS BASED ON WEB

Choi H.K., Lee B.I., Son B.H., Park S.M.

School of Information and Computer Engineering, Inje University, Korea

In this paper, we described two multi-medical telecommunication systems, i.e., image, text, and voice communication between hospitals. The systems were designed and implemented in web environments.
The procedure was carried out using the distributed object technique, CORBA. Independent of platforms and different developing languages, the CORBA-based medical communication system proved to be very useful in system development, e.g., extension and maintenance. The other system was constructed in the form of an ActiveX that could reduce the response time. With respect to maintenance cost, the designed system is of high quality.

The two developed systems were compared in terms of their architectures, as well as efficiencies. As the result of test for two systems, the response time results of the first system, which are displayed using a RGB color image $(640 \times 480)$, were as follows: time from client to CMO was $79.90 \mathrm{~ms}$; time from CMO to OMO was $40.05 \mathrm{~ms}$; time from OMO to IPO was $14.00 \mathrm{~ms}$. The response time results of the second system were follows: time from Pusan to Seoul was $0.10 \mathrm{~s}$ for a image with $106 \mathrm{k}$ size, $0.20 \mathrm{~s}$ for a image with $906 \mathrm{k}$ size and $0.40 \mathrm{~s}$ for a image with $3600 \mathrm{k}$ size. The time for transmission was proportional to the image size.

Using the proposed systems we were able to diagnose and prognosticate about the histo-pathologies of patients over the World Wide Web.

\section{4 \\ SEVEN YEARS EUROPEAN EXPERIENCE WITH A DYNAMIC TELEPATHOLOGY SYSTEM}

Clinch N. (1), Wotherspoon A. (2), Abeler V. (5), Cross S. (3), Torstenssen S. (4), Maddison J. (1), Hameed T. (1), Danielsen H. (5)

(1) Fairfield Imaging Limited, Tunbridge Wells, Kent, United Kingdom, (2) Department of Pathology, Royal Marsden Hospital, London, United Kingdom, (3) Department of Pathology, Royal Hallamshire Hospital, Sheffield, United Kingdom, (4) Department of Pathology, Kalmar County Hospital, Kalmar, Sweden, (5) Department of Pathology, Norwegian Radium Hospital, Oslo, Norway

In 1995 a decision was made to look into the possibility of building a commercial dynamic telepathology application based on diagnostic-quality digital imaging with no reliance on videocodec technology. At that time the challenge for a digital imaging system was that it be quick enough to be used in standard clinical practice for remote diagnosis and consultation.

We chose to use Internet Protocol, Tiff 6.0 and a $1024 *$ 1024, 6.8 $\square$-pixel digital camera on a laboratory microscope retrofitted with a motorised stage and focus 
drive. At that time transmission was by VSAT satellite link or by ISDN or LAN. The product was called PathSight. Version 1 had its commercial launch in a satellite link from Spitzbergen to Oslo in October 1996. A key feature of this early programme that has been retained to the present day was a "navigation map" made by decimating a montage of images in the lower power objective.

Early use of the system was for online specialist referral in histopathology. In 1998 a concordance study demonstrated reliability for remote frozen section diagnosis, and subsequently this has been one of the common uses of PathSight. A large international multi-centre concordance trial for referral and routine histopathology was completed in 1999. Attention soon shifted to the speed with which reliable routine diagnosis could be made using telepathology and a landmark study using PathSight was published by Cross et al. in 2001. At the same time an Edinburgh study of the usefulness of digital pathology presentation in over 1000 multi-disciplinary case conferences showed high acceptance from both pathologists and clinicians. Results from these studies will be presented.

In 2002, many original features remain in PathSight Version 4, although the system has now become completely modular. While still supporting one to one referral, PathSight provides one to many clinical conferencing, minimum data set reporting and virtual microscopy. The latter is a powerful extension of PathSight's old store-and-forward feature that will play a significant role in the future of telepathology. For example, one ongoing PathSight trial features automatic overnight scanning of routine histopathology caseload material in a major UK teaching hospital.

\section{5}

\section{TELECYTOLOGY PILOT PROJECT TO SUPPORT REGIONAL LABORATORY SERVICES IN EASTERN CAPE, SOUTH AFRICA. EVALUATION OF THE SYSTEM INSTALLED}

Banach L., Lancaster E., Mbalo N.

Department of Pathology, Faculty of Health Sciences, University of Transkei, Umtata, Eastern Cape, South Africa

BACKGROUND: Since 1999 South African Department of Health has embarked on national telemedicine program. St Elizabeth Hospital in Lusikisiki and Umtata General Hospital (UGH) in Umtata were chosen for pilot telecytology/telepathology project to support laboratory services in remote hospitals.

OBJECTIVE: To evaluate the system installed at $\mathrm{St}$ Elizabeth Hospital and compare with already used system (VAMSTEC, Croatia) at UGH.

METHOD: 3CCD analogue camera was used to capture images and MediRad software (LINE Imaging Systems, LCC) for storage and transfer images via 128 kbts ISDN line. Forty PAP smears with atypic and dysplastic changes were chosen and transferred. On average 4 images per slide were sent. Time of transfer was also recorded. Reviewing was performed by pathologist and diagnoses recorded and compared with those done conventionally. Satisfaction or criticism of sending and receiving pathologists was recorded.

RESULTS: The time of transfer was about 14 min per case. The quality of images was satisfactory and there was $95 \%$ concordance in the diagnosis. Criticism included quite long time of transfer and lack of "manipulation" with images on MediRad software, and also lack of export of images to other applications. Annotation of the case (history, cytology description and results) was not satisfactory.

CONCLUSION: The system installed is suitable for store-and-forward telecytology but has got limitations compared with the other system.

\section{6}

\section{TELEMEDMAIL: FREE SOFTWARE TO FACILITATE TELEMEDICINE IN DEVELOPING COUNTRIES}

Banach L. (1), Frazer H.S. (2), Jazeyeri D. (3), Szolowitz P. (3), McGraph St.J. (4), Mostafa M.G. (5)

(1) Telemedicine Unit, University of Transkei, Umtata, Eastern Cape, South Africa, (2) Children Hospital Informatics Program, Boston, MA, USA, (3) MIT Laboratory for Computer Science, Cambridge, MA, USA, (4) TUFTS University, Boston, MA, USA, (5) Anawara Diagnostic Center, Dhaka Medical College, Dhaka, Bangladesh

Telemedicine offers the potential to alleviate the severe shortage of medical specialists in developing countries. However lack of equipment and poor network connections usually rule out videoconferencing systems. This paper describes a software application to facilitate store-and-forward telemedicine by email of images from digital cameras. TeleMedMail is written in Java and allows structured text entry, image processing, image and data compression, and data encryption. 
The design, implementation, and initial evaluation in telepathology are described.

7

TELENEUROPATHOLOGY FEASIBILITY STUDY BASED ON ROBOTIC MICROSCOPY AND INTERNET

Szymas J., Wolf G., Papierz W., Jarosz B.

Department of Pathology, University of Medical Sciences, Poznan, Poland

Teleneuropathology is the practice of conducting remote neuropathological examinations with the use of telecommunication links. Because of a limited number of expert neuropathologists, some, especially smaller departments have the equipment to conduct the examination but do not have a specialist who would be able to evaluate material from the central nervous system. A teleneuropathology system directed by Java programs through a standard Internet browser was evaluated in the present study. Assessment of neurooncological cases was done by remote microscope through the "Case Study" Web page at the Department of Pathology Web site (http://ampat.amu.edu.pl). The site was used to control a remote robotic microscope, the Axioplan 2 (Zeiss), which was connected to a computer that acts as an Internet server. This feasibility study examined the diagnostic accuracy of 120 cases randomly selected from the routine diagnostic workload in Neurosurgical Pathology Laboratory in Poznan, Poland. Telepathology diagnoses were compared with conventional paraffin section diagnosis served as gold standard. The neuropathologists, operating a robotically controlled motorized microscope over the Internet individually reviewed the cases using computer workstations. Viewing times ranged from $2 \mathrm{~min} 54 \mathrm{sec}$ to $32 \mathrm{~min} 12 \mathrm{sec}$ per case. The mean diagnostic accuracy for telepathology diagnosis was of $95 \%$, with 2 of 3 observers achieving $100 \%$ diagnostic accuracy. Image quality was judged to be sufficient for a correct evaluation and the viewing times required to establish a final diagnosis by remote video microscopy were acceptable. Generally, user acceptance of robotic telepathology was good. High levels of diagnostic accuracy was achieved using robotized telemicroscope with the possibility to examine the entire histological specimen under control of the remote teleneuropathologist. On the contrary static-imaging systems appear to have levels of diagnostic accuracy which are not satisfactory for diagnostic neuropathology.
8

\section{FROZEN SECTIONS VIA TELEPATHOLOGY IN THORACIC SURGERY}

Rieck G. (1), Stoffels T. (1), Theile A. (1), Junker K. (1), Leschber G. (2), Linder A. (2), Müller K.-M. (1)

(1) Institute of Pathology, BG-Kliniken Bergmannsheil, University of Bochum, Germany, (2) Department of Thoracic Surgery, Hemer, Germany

Background: Frozen sections in thoracic surgery are necessary to distinguish benign and malignant processes followed by optimised surgical procedure.

Material and Method: We examined 76 frozen sections via telepathology using the HISTKOM-System from 53 Patients who underwent thoracic surgery procedures. The examinational tissue contains: 43 lung tumours, 15 lymph-nodes, 14 bronchus-amputates, 2 thyroids and 2 mediastinal tissues. All diagnoses have been proved by conventional microscopy.

Results: No false positive diagnose was made. 37 of the lung tumours have been diagnosed as malignant process. 5 of the lung tumours have been diagnosed as benign and one as not secure malignant process (turned at microscopy to a malignant adenocarcinoma; false negative). 5 lymph-nodes showed tumour involvement, 10 have been diagnosed as tumour-free, but at microscopy 2 turned to tumour involved lymph nodes ( 2 false negative). All 14 bronchus-amputates and both thyroids showed no malignant tumour involvement via telepathology. At final examination one papillary adenocarcinoma of the thyroid was diagnosed (false negative, sampling error). The 2 sample-tissues out of mediastinum have been tumour involved lymph nodes and thymus rests.

Conclusion: The examinations of frozen sections in thoracic surgery via telepathology is possible. In our study no false positive diagnosis was made and in $94.74 \%$ the correct diagnosis already could be made by telepathology. This method opens hospitals without possibility of frozen section examinations a better medical support to their patients and in hospitals with long distance transfers to the next institute of pathology time and money can be saved.

\section{9}

PARALLEL PROCESSINGS OF SUPER-HIGH RESOLUTION MICROSCOPIC IMAGES (SHRMIS) FOR THE USE OF THE NEXT GENERATION TELEPATHOLOGY

Tsuchihashi Y. (1), Ogino H. (2), Sakai T. (3) 
(1) Hospital Department Pathol., Kyoto Pref. Univ. Medicine, Kyoto, Japan, (2) Department Social Informatics, Kyoto Univ., Kyoto, Japan, (3) Venture Business Promotion Office, HORIBA, Ltd., Kyoto, Japan

Super-high resolution microscopic images (SHRMIs) with a pixel density of 4 millions enable pathologists to observe histologic and cytologic findings in very details in even a low power magnification and are considered very useful for pathology diagnosis. However each SHRMI bears a vast amount of information and hinders its quick processing by usual computers and its transfer by conventional net connections.

Here we introduce a new efficient method of processing a SHRMI by its parallel division into two 2 millions sub-images captured by two separate parallel-set high definition cameras on a single microscope and subsequent parallel transfer of the images on a Giga-bit ether networks and by final re-synthesis of the two subimages into a SHRMI in an original form on a superhigh resolution LCD.

The time required for the total parallel processing of an SHRMI starting from its division capturing, transferring and to reproduction to original single image form was found to be in the average 3 second and the time was considered well tolerable for the practical telepathology. Low magnification SHRMIs obtained by $\times 2$ or $\times 4$ objectives enabled pathologists to obtain simultaneous understandings to considerable degrees of characteristics of tissue structures and also of individual component cells of a tumour. Higher magnification SHRMIs gave a very fine details of nuclear chromatin patterns and also of cytoplasmic granularities of individual cells. The parallel processing method of SHRMIS will provide one of the choices for the use of the next generation telepathology diagnosis, consultation or remote pathology education.

10

\section{UNSUPERVISED HISTOLOGICAL IDENTIFICATION OF BREAST GLANDULAR AGGREGATES - APPLICATION WITHIN AUTOMATED TUMOUR RECOGNITION, TELEPATHOLOGY, AND INTELLIGENT COMPRESSION}

Leong J.W.W.-M. (1), Brady J.M. (2),

McGee J.O’D. (3)

(1) Nuffield Department of Clinical Laboratory Sciences, John Radcliffe Hospital, University of Oxford, Oxford, United Kingdom, (2) Department of Engineering Science, University of Oxford, United King- dom, (3) Nuffield Department of Clinical Medicine, John Radcliffe Hospital, University of Oxford, Oxford, United Kingdom

Recognition of glandular aggregates is a useful segmentation precursor, effectively reducing an image to a more manageable parcels for analysis. We describe a process used in an automated system designed for breast cancer recognition.

Benign glands cluster in an orderly manner while malignancy demonstrates a disordered architecture and looser clustering arrangement. Within a single digital image there may be more than one gland cluster. Alternatively, tumour may be so diffuse as to encompass the entire image. The principal difficulty with automated identification of clusters is in determining the number of clusters present. Statistical cluster analysis techniques do not perform well without some form of manual intervention and consequently these were deliberately avoided. Instead fundamentals of scale-space theory were utilised.

Using low-power breast histological images, mean nearest neighbour and variance of cell nuclei distances was found to be $100 \%$ effective in distinguishing 47 images which contained diffuse tumour and no clustering. Gaussian smoothing of the low-power image resulted in broad demarcations of glandular and stromal areas. Minimum variance quantisation effectively partitioned the image into regions determined by the average intensity value. This corresponded with glandular clusters and allowed segmentation of individual benign and malignant gland clusters in the remaining images. The broader implications of this technique lie in intelligent histological image compression and also in telepathology where prior demarcation of areas containing salient information will allow more selective data compression and transmission.

\section{1}

\section{ADICAP SERVER AT CRIHAN: SECURING CASE EXCHANGE IN DIAGNOSTIC MICROSCOPY}

Cordier J.-C., Hémet J., Martin E., Troussard X.

ADICAP (Association for Development of Computer use in Cytology and Anatomic-Pathology) - CRIHAN (Center for Research and Computer use in High Normandy), France

Needs for securized teletransmission in Pathology led to setting up a network designed specifically to suit 
Pathologists needs. The system is based upon an intercommunication server which uses standard Internet protocols. This ADICAP server is located at CRIHAN. Data format and exchange protocol has been described previously (5th EGT Congress), they are based upon a standardized document format which has been defined both by Scientific Societies and Industrial Companies.

A New series of features has been recently added:

- Answers for second opinion can be directly handled by the server and they are stored in association with the original folder. If necessary, it will be possible to ask for these document.

A free usable workstation (OpenCaseMaker) allows any pathologist to build a standardized ADICAP folder from his own images and to send it to any participant or group participating to the ADICAP network. Moreover a simplified inscription is proposed for sending or for receiving folders of images.

A dedicated services can be set up: i.e., the one developed for therapeutical protocols (see GOELAM communication). This kind of service allows handling large volume of data $(\geqslant 50 \mathrm{Mb})$ like needed with TELESLIDE (see TELESLIDE presentation) document format. This add on to the ADICAP folder allows great improvement in image representativeness very useful for telepathology efficiency.

- Finally the standard interface will be set to English so that European participation will be made easier.

Today about 700 pathologists and hematologists participate to the ADICAP network; we hope that these new features will help in expanding the secured utilization of tele-expertise in a significant manner.

\section{2}

\section{QUALITY ASSURANCE FOR CERVICAL} CANCER. A CYTOLOGICAL QUALITY CONTROL BASED ON TELESLIDE SYSTEM

Vincent S., Klossa J., Cochand-Priollet B. and the members of the committee $\mathrm{N}^{\circ} 1$ of AFAQAP

(Association Française d'Assurance de Qualité en Anatomie et Cytologie Pathologiques).

AFAQAP, France

AFAQAP is the French Association for Quality Assurance in Pathology, officially recognized by the Ministry of health. AFAQAP is composed by six committees concerning different fields in pathology: Public Health problems like colon, breast and prostate cancers as well as techniques, like immunohistochemistry or lab's organization. The committee number 1 concerning cervix cancer is the only one entirely based upon cytology. The first action of the committee concerned recommendations for the internal quality control of the Pap-smears.

An external quality control has also been organized, firstly based on sites visits by experts, then based on a CD-ROM using the ADICAP standardized image folder. The first CD-ROM included 10 cases of normal and abnormal Pap-smears chosen by the members of the committee 1 . If quality of the images was quite good, their unmoving characteristic was not satisfying. This kind of CD-ROM allows to assess the diagnostic skill of the participants but not their ability to detect an abnormal cell in between numerous normal cells.

For this reason, a TELESLIDE document format is now proposed for the next CD-ROM and will be tested. The aim of applying this technology to the CD-ROM Pap-smears quality control is to provide numerous selected areas, a full navigation follow-up and access to all magnification chosen by the producer.

This technology offers a close reproducibility with the cytopathologists routine practice and seems to be the best method for an effective diagnostic quality control.

\section{3}

\section{TELESLIDE: TECHNICAL ASPECTS}

Klossa J., Horain P.

TRIBVN and INT (National Institute of Telecommunication), France

TELESLIDE is a proposal of document format which is aimed to provide a better slide representativeness for application in the field of diagnostic microscopy.

TELESLIDE handles Wide fields of images whose size is generally $>100$ Mpixels. TRIBVN and INT (National Institute for Telecommunication) are currently developing a set of tools that will provide effective solutions for routine implementation of TELESLIDE format both for the production process and for the browsing features. We will present and discuss our technical choices.

These solutions are bases upon low cost motorized microscope, mono CCD digital camera using standard IEEE 1394 interface and digital video standard.

Data are acquired through a Bayer Matrix which means that initial signal is monochromatic digital signal and that we must pay special attention to the RGB matrixing process and to the bandwidth optimization. 
Even if high quality microscope lenses are used resulting images show a series of defects which are linked to light non uniformity over the whole field of capture. A shading correction has to be developed for achieving an homogeneous result.

Next step consists in producing a mosaic (stitching) with individual images: it should need minimum overlap, be precise not adding blur at image transition and computing time should be low. The mosaic process should also be robust in front of difficult situation like stitching different $\mathrm{z}$ images or images with nearly no distinguishable info. We are trying to improve the today process.

Last step is the sharing over digital media that need optimized compression solution. For the first TELESLIDE medical experimentation we used standard jpeg compressed tiles which allows browsing through simple HTML browser. However we are evaluating more sophisticated approach using JPEG 2000 wavelet method which could led to lower size image and which provides multi-resolution handling and differential coding for multiple $\mathrm{Z}$ images.

\section{4}

\section{TELESLIDE: BETTER SLIDE} REPRESENTATIVENESS FOR DIGITAL DIAGNOSTIC MICROSCOPY APPLICATIONS

Klossa J., Flandrin G., Hémet J.

ADICAP (Association for Developing the use of computer in Cytology and Anatomical Pathology) and TRIBVN, France

Images folders (Store and Forward), tele-microscopy (remote microscope) and Virtual Slide (Virtual Microscope) are major digital microscopic image applications. In practice, the use of images folders (including few representative images) is very widely spread but the lack of slide representativeness has become a restriction for some applications as Second Opinion, Quality Control and Frozen Sections Tele-microscopy could be an answer, but is time consuming and costly (diagnosis time is multiplied by 6 in accordance with Zeiss European study based upon $384 \mathrm{kbs}$ link) and the motorized microscope remains dedicated to a single slide.

Virtual Slide use is an alternative manner, but it needs hours to produce high resolution image of each slide (to date $4 \mathrm{~cm}^{2}$ at $\times 40$ need 28000 VGA screens).

The TELESLIDE document format could be an extension to the standardized image folder that could help to remove present limitations and would remain easy to use in routine context.

Producing a TELESLIDE document needs a routine motorized Microscope with a TELESLIDE dedicated software (see other communication TELESLIDE: technical aspects). Steps are as follows:

- Image acquisition settings are standardized.

- Screening quality scan of Region Of Interest (ROI) provides insurance for slide representativeness.

- Selected area inside the ROI are scanned at diagnostic magnification.

- Coordinates are recorded for each elementary image.

- Production of hundredth of fields takes 10 to 20 minutes.

Viewing hundreds TELESLIDE needs simple HTML browser through CD-ROM or Internet.

- Viewing interface provides full navigation follow up and access to all magnification chosen by the producer.

- TELESLIDE provides an enrichment to a standard ADICAP folder and slide representativeness avoids diagnosis induction.

- Displayed coordinates of each view allows extended interactivity between users (i.e., ask for other fields).

Today Quality control and Second opinion evaluation of TELESLIDE appear to be very promising.

\section{5}

\section{NEW DIGITAL MICROSCOPIC IMAGE APPLICATIONS ABOUT ADICAP PLATFORM FOR TELEPATHOLOGY}

Cordier J.-C., Hémet J., Martin E., Troussard X. ADICAP and CRIHAN, France

ADICAP and CRIHAN have created a universal solution to exchange medical files based on Open communications protocols (Internet) providing interoperability of the workstations available on the market. The specifications of this service have already been described during 5th EGT Congress and are published on the web (http://www.adicap.asso.fr).

Basic ADICAP service:

- Reading a folder is possible outside labs from any computer connected on the Internet: RNIS access can also be used.

- Each user has a personal account on the server and can manage his own files. 
- A file stored on the server can be accessed simultaneously by several experts.

- The management is independent (CRIHAN).

- The standardized protocol and formats(transmission and ADICAP file) have been chosen by both Scientific Societies and Industrial Companies.

- The most recent securized transmission techniques are used.

\section{Features recently added or in progress:}

- Producing files without expensive workstations: OpenCaseMaker, a free usable "on line" virtual workstation allows any user to collect digital images he produced in an ADICAP folder for any participant belonging to the service. Proposed by TRIBVN, OpenCaseMaker is located at CRIHAN.

- First inscription and connection to the server will be made easier.

- Answers for second opinion will be handled by the server and stored in association with the original folder.

\section{Perspectives:}

- Extension to other specialities (Ophthalmology, Dermatology).

- Dedicated services like the one developed for therapeutical protocols (see GOELAM communication).

- Application of medical standards and administration (security, confidentiality, authentification, traceability, statistics, archiving of medical records, DICOM compatibility, ....).

- Integration of very large files, virtual slides, wide fields (see relevant TELESLIDE presentation) which can be easily stored at CRIHAN.

- Setting the standard interface to English so that European participation will be easier.

\section{6}

\section{A PILOT TELEDIAGNOSIS REVIEWING VIA INTERNET IN THE AML-2001 THERAPEUTIC PROTOCOL}

Leymarie V. (1), Klossa J. (2), Paulin E. (2), Falkenrodt A. (1), Lioure B. (1), Harousseau J.L. (3), Reiffers J. (4), Colombat Ph. (5), Cordier J.C. (6),

Flandrin G. (7), Daliphard G. (8) and the

BGMT-GOELAMs cytologists

Laboratories and/or Departments of Hematology (1) Strasbourg University Hospital, (2) Tribvn Inc. Comp., Neuilly, (3) Nantes University Hospital, (4) Bordeaux University Hospital, (5) Tours University Hospital, (6) CRIHAN-Rouen, (7) Necker University Hospital, Paris, (8) Reims University Hospital, France
The morphological examination of blood and bone marrow stained films remains a determining first step in the diagnosis of acute myeloid leukemia. Forty cytologists working for the multicentric BGMTGOELAMs AML-2001 therapeutic protocol have set up an innovating teletransmission organization for rapid morphological diagnosis assessment.

Each participating center is equipped with a workstation numerizing and transmitting cell images in association with biological data through a standardized multimedia folder. For each newly included patient, a complete folder is sent to the CRIHAN server. Subsequently, the coordinating cytologists, informed by e-mail, pick up two review centers. These reviewers browse the multimedia folder and send back their own interpretation. The whole service is provided by the TRIBVN server, but for legal reasons the folders are stored on the ADICAP/CRIHAN server which guaranties confidentiality.

The foreseeable problems in our scheme include sending convincing images and avoiding selection bias. Initially, we defined rules for image collection. Now, we are working on TELESLIDE format for routine production of wide field images to definitively solve this crucial problem.

The AML-2001 protocol started on November 2001. Up to now 69 patients have been included. 38 folders (55\%) have been sent and 29 (42\%) were actually reviewed within an average of less than 40-days delay. A preliminary analysis shows a satisfying matching between reviewers interpretation (20/29). Our aim is to improve the reliability of diagnosis through the realtime exchange via internet. Progress in pathology is related to the application of new biological techniques and also to the impact of electronic devices affecting transfer of information and images.

\section{7}

\section{MODULARITY, OPEN SOURCE AND OPEN STANDARDS AS KEYS FOR SUCCESSFUL APPLICATION OF TELEPATHOLOGY}

Haroske G. (1), Oberholzer M. (1), Brauchli K. (2)

(1) Institute of Pathology, Dresden-Friedrichstadt General Hospital, Germany, (2) Institute of Pathology, University of Basel, Switzerland

Current telepathology is characterized by a multitude of technological solutions and logistics depending on the specific goals the users / vendors want to meet. The resulting inoperability between single applications and systems is a major obstacle for introducing as well as 
for accepting telepathology as a normal working tool for pathologists at their desks. It is the wide scale of quite different needs in telepathology that lead to the variety of different solutions.

Despite of different user needs and scenarios telepathology can be understood as a framework of basic functions with a database for image data in its core. If those functions are thought as single, relatively independent modules with clearly defined interactions, then standards can be defined both at a general and at specific levels of logistic and technological solutions. It is very important not to restrict the standardization to the technology alone, but also to agree standards for the application aspects of telepathology.

The concept of strictly modular telepathology was realized in the iPath project (http://telepath.patho.unibas. ch). With several hundred of users from quite different fields of pathology, from highly developed as well as from third world countries iPath is showing a high degree of user defined flexibility and acceptance. Around an image data base several additional modules for imaging, microscope control, administration etc. are constructed and placed in the Internet. Internet technologies provide a high level of standardization for the technological aspects. The interfaces between the modules are simple, because the functions are simple, too. The cornerstone of the concept that combines modularity and standard ability was the decision to develop all the software for the modules as open source. In that way any additional modules can be fitted to the existing ones following simple recommendations for pertaining the basic philosophy of the project.

\section{8}

\section{TELEPATHOLOGY NETWORK IN HUNGARY}

Cserneky M. (1), Szende B. (1), Vári S. (2),

Kádár A. (3), Csanaki Gy. (4), Cooke J. (4),

Cserni G. (5), Szabolcsi I. (6), Vadnay I. (7),

Szalai K. (8)

(1) 1st Department of Pathology and Experimental Cancer Research, Semmelweis University, Budapest, (2) Vári Ltd., Budapest, (3) 2nd Department of Pathology, Semmelweis University, Budapest, (4) County Hospital, Szombathely, (5) County Hospital, Kecskemét, (6) County Hospital, Székesfehérvár, (7) Ferenc Markhot County Hospital, Eger, (8) Department of Pathology, University Szeged, Hungary

Pathologists in Hungary evaluate yearly several hundred thousands of histological cases. Second opinion is needed in 5-10 per cent of cases. Application of the multimedia systems (i.e., telepathology) is inevitable for the sake of correct and fast diagnosis. The first telepathology connection in Hungary has been established between the 1st Department of Pathology and Experimental Cancer Research, Semmelweis University and the Hospital of the Ministry of Internal Affairs in Budapest. Further development occurred in the frame of various projects, supported by the EU (Interpath, Retransplant, Bepro), installing new stations in two University Institutes and six county hospitals. Electronic fixation of the images, their transmission by telephone line (ISDN) is an important feature of the multimedia system applied in telepathology. This technique is easily available. The telepathology system enables us

- to decrease the duration of hospitalization

- to decrease the time for anesthesia in case of frozen sections

- to make possible professional and fast histological diagnosis for surgical departments if the hospital is lacking pathologist

- to ensure quality control and facilitate permanent postgradual teaching first of all by establishing data bases of histological images.

The system used by as is apt for evaluation of frozen or paraffin-embedded sections, immunhistochemical and cytologic samples, supplemented with transmission of macroscopic pictures. Our experience on bilateral consultations underlines the importance of telepathology. The telepathology system established in Hungary joined successfully to the telepathology network in the EU (Berlin, Grenoble).

\section{9}

\section{THE DEVELOPMENT OF AN INTERNET BASED, PATHOLOGY TRAINING TOOL}

Johnston D.J. (1), Costello S.P. (1), Dervan P.A. (2), O'Shea D.G. (1)

(1) Medical Informatics Group, School of Biotechnology Dublin City University, Ireland, (2) Department of Pathology, Mater Misericordiae Hospital, Dublin, Ireland

The Virtual Pathology Slide is a unique interactive Internet diagnostic tool developed by the Medical Informatics group in Dublin City University. The VPS is capable of digitising a complete slide at $40 \times$ and presenting it for examination in real-time on an Internet enabled PC. The slide may be viewed at a variety of magnifications and movement in X \& Y Cartesian planes, 
at a given magnification, is available. As the complete slide is digitised, examination of the slide is not restricted to pre-selected areas. As an examination is performed online, information pertaining to the diagnostic process may be tracked and recorded. Locations of regions of interest, duration spent examining these regions and comments relating to specific areas of the slide are recorded and stored.

Using this behavioural information and the technology developed for the VPS, a comprehensive training tool has been developed. This tool comprises two specific functions:

1. Replay of examinations in real-time.

2. Comparative analysis of examination data.

The VPS is capable of replicating the diagnostic trace of an examination in real-time, by presenting, in order, regions of interest examined and the time taken to examine these regions. As this technology is of particular relevance to pathologist training, it is our intention to create a library of experienced pathologist's examinations of a selection of training and advanced cases.

The practice of microscopic diagnosis has always been a subjective science, which is based upon the ability of the human brain to recognise patterns based on experience. Interobserver variability is unavoidable. Analytical comparison of tracking data recorded during examinations on the VPS allows variability in decisionmaking processes to be observed and explained in an objective manor. This ability to evaluate the efficiency of diagnostic processes is of considerable value to both training and diagnostic quality.

\section{0}

\section{MODULAR INFRASTRUCTURE FOR TELEPATHOLOGY}

Brauchli K., Oberholzer M.

Department of Pathology, University of Basel, Switzerland

Traditionally Telepathology was mainly focused on remote microscopy. With the introduction of Internet and Email in recent years, store-and-forward telepathology internet sites have emerged providing remote consultations to a larger community. To facilitate the use of modern information and communication technology for telepathology we have developed an open source telepathology framework which allows implementations of a wide range of web based telepathology applications from case databases for teaching, spe- cialist consultations to real time remote microscopy (http://ipath.sourceforge.net).

The system is designed in a modular way. At the core is a database which manages case information and user authentication. The main access to the database is provided in form of a web interface but additional interfaces can be implemented as separate modules. Modules for real time remote microscopy, case import over email and and $\mathrm{xml}$ reporting module already exist.

With the use of XML, declared interfaces and freely available software telepathology consultation is no longer limited to single websites. Thus, networks for telepathology can easily be implemented on different levels ranging from intra-hospital services like image archive and tumor boards, regional services like remote frozen section diagnosis and remote laboratory assistance to international specialist consultations. Pathologists can easily be active in many different telepathology networks and refer cases from one network to another if needed.

\section{1}

\section{TRIDEM: PRODUCTION OF CONSENSUAL CASES IN PATHOLOGY USING TELESLIDE OVER INTERNET}

Zapletal E. (1), Le Bozec C. $(1,2)$,

Guinebretière J.M. (3), Jaulent M.C. (1), Hemet J. (4)

(1) ERM 0202 INSERM, (2) Georges Pompidou University Hospital, (3) Gustave Roussy Institute, France, (4) ADICAP, France

Poor intra- and inter-observer diagnosis agreement in pathology is largely reported in the literature. Previous studies have noted that consensus sessions can reduce observer variation in standardizing classification systems. Internet may make both the derivation and the dissemination of consensus easier and more efficient. Two main unsolved issues for consensus over Internet are to provide sufficient high quality images and to compute description comparisons.

We present TRIDEM, an Internet platform designed to assess observer variation in histopathologic descriptions. TRIDEM combines two modules: a module producing teleslides to provide sufficient images to pathologists and a consensus module developed to compute observer agreement on descriptions. This tool allows pathologists to label precise regions of interest in images and to derive semi-automatically multi-experts consensual descriptions of cases from initial descriptions. 
A pilot study was carried out to assess the performance of the consensus module. A senior and junior pathologist labelled 83 images of 53 benign and malignant cases of breast pathology. Images were assessed twice within a 1-month period by the junior pathologist.

Based on the computed comparison of descriptions, final joint descriptions were rapidly achieved (median time of $2 \mathrm{mn} 04$ s per case) and were significantly more complete than initial descriptions.

We conclude that teleslide technology, providing representative document format compared to static image folder, allows experts to derive consensus over Internet with sufficient images. Furthermore a computerized system automatically comparing histopathologic descriptions of a panel of pathologists makes the consensus more efficient and offers the perspective to build useful image databases with unambiguous and consensual descriptions of cases.

\section{2}

\section{ENABLING BEST PRACTICE IN ONCOLOGY (BEPRO)}

Debreczeni K., Vári S.M.D., Cserneky M.

Semmelweis University Budapest, Hungary

Introduction: The aim of the $\mathrm{BePrO}$ project, sponsored by the European Commission (IST-2000-25252), is to demonstrate best practice in action by using innovative information and computer technology (ICT) applications in Oncology.

Results: A European network and four medical reference platforms were created in France, the United Kingdom, The Netherlands and Hungary, each at the heart of a professional network, where the information and communication technologies for health have been integrated in daily practice. Apart from enabling sharing experience and consultation, the program implements continuous medical education as well. The electronic multimedia textbook provides information on breast and lung cancer. Up-to-date related articles and guidelines supplement the presented scientific material and a rich collection of macroscopic and microscopic pictures makes the electronic textbook interactive and more colourful. Besides, various interactive case presentations discuss interesting and instructive cases on both fields providing decision-making challenges and the possibility to test knowledge on breast and lung cancer. A complex and logical link system to the electronic textbook and within the textbook makes the education more effective.
Conclusions: The BePrO project carried out safe integration of medical best practices in several respects: reference diagnostics, consensus on electronic forms and remote data entry to epidemiological centres and clinical trials coordinators, guidelines and continuous medical education.

\section{3}

\section{LARGE-SCALE QUANTITATIVE ANALYSIS OF} CERVICAL INTRA_EPITHELIAL NEOPLASIA

Guillaud M. (1), MacAulay C. (1), Follen M. (2),

Richards-Kortum R. (3), Alexeenko T. (2),

Valdizan-Garcia M. (2), Boiko I. (4), Carraro A. (1), Korbelic J. (1), Haskins D. (1), Malpica A. (2), Staekel G.A. (2), Van Nierkerk D. (5), Matisic J. (5)

(1) BC Cancer Research Centre, Cancer Imaging department, (2) UT MD Anderson Cancer Centre, (3) University of Texas, Department of Electrical and Computer Engineering, (4) UT Medical School, (5) BC Cancer Agency-Department of Pathology, USA

Histological grading of Cervical Intra-epithelial lesions (CIN) remains a subjective task, resulting in inter-and intra-observer variability and poor reproducibility of the diagnosis of cervical lesions. This study presents some results obtained from the quantitative analysis of intra-epithelial lesions of the cervix using a semi-automatic image cytometer. The objective was to extract information at the tissue level, by measuring changes in the spatial organisation of cells within the diagnostic area, and at the cellular level by measuring changes in the morphology and the texture of the nuclei. More than 1500 biopsies were obtained from more than 500 patients enrolled in a screening and diagnostic trial for the detection of SILs of the uterine cervix using fluorescence/reflection spectroscopy. All the biopsies were read at least twice. Discrepancies between the first and the second reading were resolved by a consensus review and a final diagnosis was assigned The diagnostic areas were mapped by an experienced pathologist and biopsies were classified into 5 groups: normal biopsies, koilocytosis, CIN I, CIN II, and CIN III/CIS. Four microns sections were stained with a stoichiometric DNA staining. Using the Cyto-Savant imaging system, automatic extraction of the centres of all nuclei (architecture analysis), and semi-automatic segmentation of intact, in-focus nuclei (cytometric analysis) were performed within a pre-defined diagnostic area. About 25 features describing the global architecture of the epithelium were measuring and combined to construct an ar- 
chitectural score. Around 110 cytometric features were combined into eighth cytometric scores, each measuring a specific changes of the preneoplastic process at the cellular level. Correlations between these scores and the pathology grading will be described. A combination of all these features was used to create an Histometric space. Finally we will describe how different approaches - combining conventional pathology and quantitative scores - could improve the classification of cervical intra-epithelial lesions.

\section{4}

\section{EARLY LUNG CANCER DETECTION USING IMAGE CYTOMETRY: WHAT ARE THE CHALLENGES?}

Doudkine A., MacAulay C., Garner D., Palcic B.

Cancer Prevention Centres Inc., Perceptronix Medical Inc., and British Columbia Cancer Agency, Vancouver, Canada

Lung cancer is the number one cancer killer in both men and women in North America. In general, lung cancer is discovered in patient at a very late stage for which there are no effective cures. In contrast, if lung cancer is discovered at stage 0 or 1 , it can be cured with great probability of long-term survival. We have been exploring the thesis that quantitative sputum cytology based on a fully automated, high resolution image cytometry could be used for detecting lung cancer at its early stage.

In very early lung cancers there are seldom any cancerous cells exfoliated in the sputum. Because of this, conventional sputum cytology has poor sensitivity, generally below $15 \%$ for stage 0 and 1 lung cancers. We proposed that measuring the Malignancy Associated Changes (MAC) in cells exfoliated from noncancerous lung tissue could be used for early cancer detection. These changes are manifested in very small, but measurable population shifts of selected nuclear features. We also propose that epithelial cells extracted from other tissues of lung cancer patients can express MAC and therefore potentially provide complimentary diagnostic information. This paper will discuss several aspects of imaging of MAC and methods that may help minimizing the associated measurement errors, such as normalization/correction against staining variations and improvement in illumination of imaging system. Our results on several hundred sputum samples, as well as samples from other tissue sites, using exclusively normal appearing cells, indicate the possibility of achieving an $80 \%$ accuracy discriminating subjects inflicted with early lung cancer versus those of equivalent, high risk population of individuals without lung cancer.

\section{5}

\section{THE USE OF AUTOMATED QUANTITATIVE SPUTUM CYTOLOGY FOR LUNG CANCER DIAGNOSIS}

Kemp R., Turic B., Palcic B.

Perceptronix Medical Inc., USA

Lung cancer is a leading cause of death, accounting for $30 \%$ of cancer deaths in developed countries. Despite the advances made in cancer treatment, the five-year survival rate for all people diagnosed with lung cancer is less than $15 \%$. This is largely due to the fact that approximately $85 \%$ of people diagnosed with lung carcinoma (in the United States) have already advanced stages of the disease. Patient prognosis is strongly dependent on how early it is detected. The five-year survival rate of early stage cancer diagnoses is $>70 \%$ while the rate for later stage cancer diagnoses is $<10 \%$. We are conducting a multi-center field trial of an automated quantitative sputum cytology (QSC) system for the early detection of lung cancer. In this system, lung sputum samples are prepared as Feulgen stained slides for automated image analysis. These slides will typically contain several thousand exfoliated epithelial cells whose nuclei can be examined. Morphometric, photometric and texture features are calculated for the images, and different cell subpopulations are identified within the sample. The population statistics of the different cell types are combined to produce a single probability of malignancy for the sample.

In our current study of more than 1000 high-risk individuals, the performance of conventional sputum cytology and QSC are being compared. Initial results indicate that is possible to increase diagnosis sensitivity by a factor of 3-4 at a reasonable loss of specificity for detecting early, operable lung cancers. The nature of the test allows us to optimize the specificity/sensitivity operating point based on the target population.

\section{6}

\section{TOWARDS SCREENING OF WHOLE LYMPH} NODES FOR DETECTING OCCULT CELLS USING LOW RESOLUTION IMAGE ANALYSIS

Tanke H.J. (1), Mesker W.E. (1), Sloos W.C.R. (1), Morreau J. (3), Doekhie F.S. (2),

Tollenaar R.A.E.M. (2), Vrolijk J. (1) 
(1) Departments of Molecular Cell Biology, (2) Surgery, (3) Pathology, Leiden University Medical Center, Leiden, The Netherlands

Thus far, RT-PCR against carcino-embryonic antigen (CEA) messenger RNA is a promising but not complete sensitive approach to detect occult cells in histopathologically assessed negative lymph nodes from patients with colorectal cancer (CRC). To improve the detection of occult cells in patients with CRC multiple sections $(n=50)$ throughout the lymph node were immunohistochemically stained and analyzed by applying low resolution image analysis (flatbed scanning) for semi-automated detection of cytokeratin (CK) positive stained cells.

The sensitivity of this approach was demonstrated for 20 patients with stage II colorectal cancer and compared with RT-PCR regarding the detection of clinically assessed recurrence of disease within 10 years. Cytokeratin positive cells were detected in all patients $(n=6 ; 100 \%)$ with recurrence, versus 5 patients $(83 \%)$ by RT-PCR. From patients $(n=14)$ who did not develop a recurrence, eight $(57 \%)$ had positive lymph nodes. In all patients with recurrence we visually identified at least one group of $\mathrm{CK}^{+}$cells $(=2$ cells).

The use of IHC-automated imaging was demonstrated to be more sensitive but less specific than conventional histopathology or RT-PCR. Further characterization of the detected cells by additional markers or by molecular analysis after laser microdissection is considered essential to identify patients that might develop recurrence.

\section{7}

\section{MULTIPARAMETRIC QUANTITATIVE INVESTIGATIONS ON THYROID NEOPLASMS. IMPROVEMENT OF DISCRIMINATION IN LESIONS OF UNCERTAIN CLASSIFICATION}

Cavallari V. (1), Albiero F., Arena G., Cavaliere R., Cicciarello R., Costa G., Iemmo R., Gagliardi M.E., Torre V., Losa G. (2)

(1) Inst. of Ultrastructural Pathology, University of Messina, Italy, (2) Istituto di Studi Scientifici Interdisciplinari, Locarno, Switzerland

A group of 236 thyroid nodules collected from total thyroidectomy specimens was analysed utilizing a multiparametric quantitative protocol. 71 colloid goiters, 21 hyperplastic goiters, 20 focal lymphocytic thy- roiditis, 22 Hashimoto's thyroiditis, 55 follicular adenomas, 5 Hürthle cell adenomas and 42 differentiated thyroid carcinomas were diagnosed according with the WHO's guidelines.

Each nodular lesion was submitted to flow-cytometry for ploidy and S-phase assessment; a set of geometric and densitometric parameters was also derived from thyroid cell nuclei and basal laminas sampled at electron microscopy.

The combination of cytometric and geometric descriptors allowed for the correct discrimination of a significant percentage of malignant lesions (40/42). The majority of hyperplastic nodular goiters $(15 / 21)$ were also discriminated from follicular adenomas.

Nevertheless, a relatively small group of lesions with a mirofollicular pattern remained of uncertain classification. Firstly, some multicentric nodular lesions without a distinct thickened capsule, classified as "hyperplastic nodules", exhibited quantitative patterns similar to the follicular adenoma group. On the other hand, capsulated lesions classified as histologically benign displayed quantitative patterns overlapping with the carcinoma group.

The fractal analysis of nuclear profiles sampled at the final magnification of 18100 allowed for discrimination of an additional group of lesions consisting with preinvasive carcinomas.

The stepwise regression analysis demonstrated a highly descriptive power of basal lamina geometric parameters, including the index of complexity, the presence of aneuploidy associated with a high percentage of cells in S-phase and, finally, the fractal dimension of the nuclear membrane.

The routinary assessment of such parameters is fast and inexpensive, involving the sampling of digital images directly from the electron microscope and the automatic outlining of the regions of interest. This allows for the identification of a group of histologically benign follicular lesions in which a more careful postoperatory follow-up (including the search for occult metastases and/or thyroid residuals) is required.

\section{8}

COLOUR IMAGE PROCESSING AND

PATTERN RECOGNITION IN THE

VISUALISATION OF BASEMENT MEMBRANE

- APPLICATION IN DISTINGUISHING MALIGNANT FROM BENIGN GLANDULAR LESIONS

Leong J.F.W.-M. (1), Leong A.S.-Y. (2), Brady J.M. (3), McGee J.O'D. (4) 
(1) Nuffield Department of Clinical Laboratory Sciences, John Radcliffe Hospital, University of Oxford, Oxford, United Kingdom, (2) Discipline of Anatomical Pathology, John Hunter Hospital, Newcastle, NSW, Australia, (3) Department of Engineering Science, University of Oxford, United Kingdom, (4) Nuffield Department of Clinical Medicine, John Radcliffe Hospital, University of Oxford, Oxford, United Kingdom

In histological examination, the absence of basal lamina is a useful feature in distinguishing invasive malignancy from benign or in situ lesions. It forms part of the basement membrane, a significant barrier that all epithelial malignancies must breach in order to invade stroma. Unfortunately, destruction of basement membrane is not always possible to assess on routine haematoxylin and eosin sections. Pathologists have instead relied upon immunohistochemistry to stain for components of the basal lamina such as laminin or type IV collagen.

We employed an original combination of colour image processing and pattern recognition techniques in an attempt to accentuate the collagenous stroma surrounding glands, which approximates the basement membrane. Digital images were captured at $40 \times$ objective magnification. The image set examined comprised 77 images of benign glandular aggregates ( 26 demonstrating sclerosing adenosis), 57 tubular carcinoma images, and 39 images demonstrating ductal carcinoma in situ. Additionally, selected tissue blocks of benign and malignant breast were stained using immunohistochemical protocols for type IV collagen and alpha-smooth muscle actin for comparison.

Distinct differences in the pattern derived through image processing were found between benign and invasive lesions and also between in situ and malignant. In isolation, this method demonstrated a sensitivity of 0.96 , specificity 0.89 and positive predictive value of 0.92 for distinguishing malignancy.

Compared with immunostaining, this method is cheaper and faster although is unable to provide the same degree of accuracy. However, as part of a larger system for automated tumour recognition, it performs a significant function.

29

ARE HIGHER ORDER ESTIMATES OF SHANNON'S ENTROPY IMPORTANT FOR IMAGE ANALYSIS?

Metze K. (1), Adam R.L. (1), Leite N.J. (2)
(1) Lab. of Experimental Pathology, Center of Experimental Medicine and Surgery, State University of Campinas, Brazil, (2) Institute of Computing, State University of Campinas, Campinas-SP, Brazil

Usually the first or second order estimates of entropy are used for the quantification of the information content of images. The aim of our investigation was to find out whether higher order estimates of Shannon's entropy could contribute to image analysis of nuclei. As a biological model we chose the chromatin changes in rat cardiomyocytes during physiological development, examining digitalized images of hematoxylin stained nuclei (100 cells per animal). Ninety animals ageing between 19 days of gestation and 60 days after birth entered the study. The absolute (ASE) and normalized (NSE) Shannon's entropies were calculated from theco-occurrence matrices of the 1 st up to the 20 th order. The entropy estimates increased with the matrix order, converging to final values, which were achieved in $96.4 \%$ of the cells in the 8th order cooccurrence matrix. All entropies showed negative correlations with the age of the animals, thus reflecting increasing anisotropy of the heart. With increasing matrix order, the absolute values of the ASE correlation coefficients decreased, whereas the NSE correlation coefficients increased until the 6th order and than decreased. In a multiple linear regression model with age as the dependent value, the 1st order ASE and 1st order NSE were the most important variables, whereas higher order entropies gave only small contributions to the Rsquare value.

Similar results were obtained with a linear discriminant analysis. Therefore we think that in our biological model higher order estimates of entropy are of limited importance for the characterization of texture. Supported by FAEP, FAPESP, CNPQ.

\section{0}

\section{NO CORRELATION BETWEEN CHROMOSOMAL CHANGES AND CLINICAL OUTCOME IN 56 LARYNX AND PHARYNX SQUAMOUS CELL CARCINOMA}

Hermsen M.A.J.A. (1), Alonso Guervós M. (2), Meijer G.A. (1), Baak J.P.A. (1), van Diest P.J. (1), Alvarez Marcos C. (3), Sampedro A. (2)

(1) Department of Pathology, Free University Hospital, Amsterdam, The Netherlands, (2) Cytometry Service, University of Oviedo, Oviedo, Spain, (3) Department of Otolaringology, Valle del Nalón Hospital, Oviedo, Spain 
Chromosomal gains and losses in 34 larynx and 22 pharynx primary untreated carcinomas were investigated with comparative genomic hybridization (CGH) and results were related to a series of clinical parameters and follow-up information. All patients were mine workers with a history of tobacco and alcohol use. Nine tumours were stage I, 13 stage II, 9 stage III, and 25 stage IV. Histologic grade was well differentiated in 17 cases, moderate in 28, and poor in 11 . Twenty-six cases were lymph node positive. The average follow-up time was 25,5 months.

Flow cytometric analysis showed 18 out of 50 cases to be DNA diploid and 32 aneuploid. CGH analysis on average detected 11.1 gains and 8.5 losses. The major chromosome arms showing gains were (in decreasing order): $3 \mathrm{q}, 7 \mathrm{q}, 8 \mathrm{q}, 5 \mathrm{p}, 11 \mathrm{q} 13,17 \mathrm{q}$ and $18 \mathrm{p}$, and losses at $3 \mathrm{p}, 11 \mathrm{qter}, 4 \mathrm{p}, 18 \mathrm{q}$, and $5 \mathrm{q}$. Thirty-five tumours contained high level amplifications, with an average of 2.3 per case. The segments most frequently amplified were 3q26-qter (20 cases), 11q13 ( 7 cases), 11q22 (5 cases), $3 q 12-13$ (5 cases), 18p11.3 (5 cases), and 18q11.2 (5 cases). High level amplifications occurred more frequently in aneuploid cases than in diploid cases. No differences in chromosome abnormalities between the larynx and pharynx cases were found.

All six amplifications at 11q13 occurred in lymph node positive cases, all of these were stage IV tumors. However, although tumor stage and lymph node status correlated with poor clinical outcome, amplification 11q13 did not. In fact, not one specific chromosomal abnormality correlated with clinical outcome, as they did not with tumor stage, lymph node status, DNA ploidy, or degree of differentiation. These surprising results seems to indicate that genetic alterations at the level of chromosomes can not be used as prognosticators in invasive larynx and pharynx squamous cell carcinomas.

\section{1}

\section{PHOTODIAGNOSIS OF HPV INFECTIONS THROUGH MULTISPECTRAL IMAGE SPECTROSCOPY}

Stefanaki I. (1), Themelis G. (3), Panayiotides J.G. (2), Balas K. (3), Toska A. (1)

Departments of Dermatology (1) and Pathology (2) University of Crete Medical School, (3) Institute of Electronic Structure and Laser, Foundation for Research and Technology - Hellas (FORTH), Greece

A big percentage of the sexually active population is infected by one or more Human Papilloma Virus
(HPV) types. However, the number of patients presenting with clinically overt lesions (condylomata acuminata) represent only the tip of the iceberg, since most genital tract HPV infections are subclinical or latent. Consequently, clinical examination underestimates the extent of HPV infection, whereas in vitro diagnostic methods are expensive and time consuming. HPV16, 18 and other HPV types have been detected frequently in carcinomas of the cervix, vulva, penis and anus.

The aim of this study was the in vivo detection and staging of HPV infection of anogenital area by quantitative assessment of the acetic acid-induced temporal and spatial alterations in the light-scattering properties of HPV-infected skin. For this reason a multispectral imaging system has been employed, which performs time-lapse imaging and enables the calculation and display of the kinetics of the provoked alterations in any point within the examined area. Lesions of anogenital area with clinical diagnosis of condylomata acuminata were studied. The evaluation of acetic acid-tissue interaction kinetics revealed quantitative parameters, containing information about the magnitude and duration of the provoked alterations. The histology evaluation and polymerase chain reaction performed, showed that the method contains specific diagnostic information, since it enables the differentiation between subclinical lesions, clinical condylomata acuminata and squamous cell carcinomas developing on condylomata. It also allows the exact localization of lesions and delineates their borders.

\section{2}

\section{COLORECTAL ADENOMA TO CARCINOMA PROGRESSION FOLLOWS MULTIPLE PATHWAYS OF CHROMOSOMAL INSTABILITY}

Meijer G. (1), Hermsen M. (1), Postma C. (1), Baak J. (1), Weiss M. (2), Rapallo A. (3), Sciutto A. (3), Roemen G. (5), Arends J.-W. (5), Williams R. (4), Giaretti W. (3), de Goeij A. (5)

(1) Departments Pathology and (2) Gastroenterology, VU University Medical Center, Amsterdam, The Netherlands (present address JB: Central Hospital of Rogaland, Stavanger, Norway), (3) Lab. Biophysics and Cytometry, National Institute for Cancer Research, Genoa, Italy, (4) Department Pathology, University of Melbourne, Australia, (5) Department Pathology, University Maastricht, The Netherlands

Current models of colorectal adenoma to carcinoma progression do not fully reflect the genetic heterogene- 
ity and complexity of the disease. The aim of the present study was to identify genetic changes discriminating adenomas that have progressed to carcinoma from adenomas that have not progressed, and to refine the current genetic models of colorectal adenoma to carcinoma progression, based on a genome-wide analysis of chromosomal aberrations.

Sixty-six non-progressed colorectal adenomas, 46 progressed adenomas (malignant polyps), and 36 colorectal carcinomas were screened for chromosomal aberrations by $\mathrm{CGH}$, and for mutations in the APC and Kras gene. Data analysis focused on cancer-associated genetic changes in adenomas.

Accumulation of losses in 8p21-pter, 15q11-q21, $17 \mathrm{p} 12-13$ and $18 \mathrm{q} 12-21$, and gains in 8q23-qter, 13q14-31 and 20q13, were strongly associated with adenoma to carcinoma progression, independent of the degree of dysplasia. Hierarchical cluster analysis demonstrated the presence of three distinct subgroups of adenomas, characterized by unique combinations of genetic aberrations in the adenomas $(17 \mathrm{p}$ loss and $\mathrm{K}$ ras mutation, $8 \mathrm{q}$ and $13 \mathrm{q}$ gain, and $18 \mathrm{q}$ loss and $20 \mathrm{q}$ gain, respectively).

The presence of two or more of the above mentioned seven chromosomal changes was associated with progressed colorectal adenomas and colorectal cancer. In addition, evidence was found that these chromosomal abnormalities occurred in specific combinations of a few abnormalities, rather than as a mere accumulation of events, indicating the existence of multiple independent chromosomal instability pathways of colorectal cancer progression.

\section{3}

\section{TEXTURE OF DERMAL ELASTOTIC TISSUE IN PATIENTS WITH DIFFERENT PHOTOTYPES}

Metze K., Gomes Neto A., Adam R.L., Gomes A.A., Leite N.J., Souza E.M., Cintra M.L.

Faculty of Medicine and Computing Institute, State University of Campinas, Brazil

Fitzpatricks's classification of the phototype considers burning and tanning responses to sun exposure and is an important predictive factor for the risk of skin cancer. There are few studies comparing dermal changes between different phototypes. The aim of our study was to investigate the texture of elastotic dermal tissue in heavily of partly sun-exposed areas of patients with phototypes I to III. It was based on 57 outdoor workers aged above 50 years, who signed an informed consent. Three-mm punch biopsies of the sun-exposed dorsal side of the forearm, and the partially sun exposed inner part of the arm were performed. Biopsies were routinely paraffin embedded and 5 micra sections stained for elastic fibers according to Weigert. A preliminary analysis on optical microscopic level could not demonstrate any difference between the three phototypes. Digitalized images were acquired from the superficial part of reticular dermis. Haralick texture features derived from the co-occurrence matrix of the original image and the Fast Fourier Transform (FFT) images were determined, as well as the coefficients of the spatial spectrum of the FFT.

Many textural and spectral parameters such as entropy, inertia and cluster proeminence showed significant differences between dermal tissue of the forearm and arm in all phototypes (Wilcoxon test). Comparing the different phototypes, textural features such as local homogeneity, inverse difference moment, and peak transition probability, showed significant Spearman correlations with the phototype in less exposed dermal tissue. Significant correlation coefficients were also found, but lower in forearm dermal tissue.

Texture analysis of digitalized histologic images is able to detect differences of elastotic dermal tissue between different phototypes, regardless of the topography, which cannot be objectified by conventional microscopic analysis.

Furthermore our results suggest that comparative studies should not be done in heavily sun exposed areas. Supported by FAEP, FAPESP, CNPq, CAPES.

\section{4}

\section{DIFFERENTIATION OF KELOID AND HYPERTROPHIC SCAR BY TEXTURE ANALYSIS}

Metze K., Silva P.V.V.T., Adam R.L., Cintra M.L., Leite N.J.

State University of Campinas and Catholic University of Campinas, Brazil

Keloid and hypertrophic scar, two types of excessive scarring, each with peculiar clinical behaviour, require different therapeutic approaches, but may be confused because of morphological similarities. The aim of our study was to investigate whether texture analysis could help to distinguish both lesions. Twenty-two keloids and 12 hypertrophic scars entered the study. In each case the diagnosis had been obtained by two observers based on histologic examination of HE and Masson's trichrome stained routine sections of paraffin embed- 
ded material as well as on clinical data. Image analysis was performed on 20 digitalized images per case.

Haralick texture features derived from the co-occurrence matrix of the original image and the Fast Fourier Transform (FFT) images were determined. Furthermore, by interpreting each pixel of the FFT images as a vector (with its length determined by its gray value and the direction defined by its position relative to the center) we calculated the resulting vectors for different frequency ranges, which can be interpreted as an estimation of anisotropy.

Images of keloids were generally more homogeneous in terms of the gray levels with less entropy than hypertrophic scars. Both for high and low spatial frequencies, keloids revealed larger resulting vectors, indicating more pronounced anisotropy.

Finally in a linear discriminant analysis it was possible to classify correctly nearly $90 \%$ of the cases by four texture parameters.

In summary texture analysis parameters can be helpful for the differentiation between keloids and hypertrophic scars.

Supported by FAEP, FAPESP, CNPq.

\section{5}

TEXTURE ANALYSIS OF RESECTION MARGINS AS A TOOL FOR DECIDING ON POSTOPERATIVE IRRADIATION FOR EARLY STAGE ORAL CANCER

Sudbø J., van Helvoirt R., Bryne M., Reith A., Sudb $\varnothing$, A.

Departments of Medical oncology and radiotherapy and Pathology, The Norwegian Radium Hospital, Institute of Oral Biology, University of Oslo, Norway, Norwegian University of Science and Technology, Trondheim, Norway

Free resection margins are taken to indicate a reduced need for radiotherapy after tumor resection, but even patients with free resection margins experience relapses after surgery.

In 223 patients with early stage oral cancer, numerical values of 2 structural features were computed in 4 different groups (patients that received postoperative irradiation, with and without recurrence of cancer, and patients that had not received postoperative irradiation, with and without recurrence of cancer). Computed values from 111 patients with relapse (68 underwent surgery alone, 53 received additional radiotherapy) were compared to those from 112 patients without relapse (35 patients underwent surgery alone,
67 received additional radiotherapy). Computed values from 68 patients that only underwent surgery alone and had a relapse were compared to those from 53 patients that also received postoperative radiotherapy and had a relapse.

Among 103 patients that did not receive radiotherapy, 68 patients with relapse, had significantly different structural feature values of DEL-av as compared to 35 patients without relapse (median value 24.8, range 23.9 to 25.7 , versus 27.1 , range 25.9 to 28.2 in the group without relapse, respectively, $p=0.005)$. Among 120 patients that received postoperative radiotherapy, 53 patients with relapse, had significantly different structural feature values of DEL-av as compared to the 67 patients without relapse (median value 25.1, range 23.9 to 26.1 , versus 27.4 , range 25.5 to 28.1 , respectively, $p=0.01) .68$ patients that underwent surgery alone and had a relapse, had significantly different structural feature values of ELH-av as compared to 35 patients without relapse (median value 0.51 , range 0.39 to 0.67 , versus 0.29 , range 0.21 to 0.38 , respectively, $p=0.01) .53$ patients that did receive postoperative irradiation but had a relapse, had significantly different structural feature values of ELH-av as compared to 67 patients without relapse (median value 0.53 , range 0.41 to 0.67 , versus 0.31 , range 0.22 to 0.37 , respectively, $p=0.01)$.

Texture analysis of tissue architecture in resection margins indicates which patients may profit from postoperative radiotherapy.

\section{6}

\section{A COMPACT SET OF ADAPTIVE TEXTURE FEATURES FROM COOCCURRENCE OF GRAY LEVEL RUN LENGTH MATRICES}

Albregtsen F. (1), Nielsen B. (1,2),

Danielsen H.E. (2,3)

(1) Department of Informatics, University of Oslo, Norway, (2) Division of Digital Pathology, Norwegian Radium Hospital, Norway, (3) Division of Genomic Medicine, University of Sheffield, England

Gray Level Cooccurrence Matrix (GLCM) based approaches to the analysis of visual textures has demonstrated a number of successful implementations. However, a large number of GLCM accumulations, using different spatial displacement parameters, are needed before the geometric properties of texels can be estimated.

The strength of the Gray Level Run Length Matrix (GLRLM) approach is that it characterizes the spatial 
interrelationship between connected and colinear pixels that are so similar that they form "gray level runs". Its weakness is that it discards information on the probabilities of transitions between gray levels.

In the present study we form a small set of statistical texture features. First we introduce the 4D Cooccurrence of Gray Level Run Length Matrix, based on neighboring gray level runs. Secondly, we use its associated 2D sum and difference matrices. Thirdly, we eliminate the classical step of pre-defined feature extraction. Adaptive texture features are obtained by using squared Mahalanobis class distance matrix values as weights in a summation within the $2 \mathrm{D}$ sum and difference matrix elements. Finally, we use the disjoint positive/negative parts of the class difference matrices as the domains of the weighted summation.

The resulting small set of adaptive texture features has been applied to the 45 most difficult Brodatz texture pairs, as well as to the peripheral parts of about 2000 mouse liver cell nuclei from two classes, normal and premalignant, and the classification results have been compared to that of classical features. The method was also applied to 10000 cell nuclei images taken from 40 patients of two classes (good and bad prognosis) of early ovarian cancer.

\section{7}

\section{STATISTICAL TEXTURE ANALYSIS - ROBUSTNESS VERSUS NUMBER OF GRAY LEVELS}

Nielsen B. (1,2), Albregtsen F. (1),

Danielsen H.E. (2,3)

(1) Department of Informatics, University of Oslo, Norway, (2) Division of Digital Pathology, Norwegian Radium Hospital, (3) Division of Genomic Medicine, University of Sheffield, England

The gray level cooccurrence matrix (GLCM) method is one of the most powerful and frequently used methods of statistical texture analysis. Each element $P(i, j)$ of the matrix contains an estimate of the joint probability of gray levels $i$ and $j$ at a given pixel distance. In diagnostic and prognostic work on cell nuclei, orientation isotropy is assumed. The size of the square matrix is given by the number of gray levels used, $\mathrm{G}$.

As we are going to extract features from the $\mathrm{G} \times \mathrm{G}$ matrix, we have to assume that the matrix faithfully represents the second order statistics of the image. This requires that the image contains a sufficient number of pixels, so that adding or subtracting a few pixels does not affect the outcome of the computation of the fea- tures. If the image is relatively small, we may have to reduce the number of gray levels in the image, possibly wasting information.

It is of interest to find the optimum number of gray levels in the generation of textural features. $G$ may be increased either by accumulating an average matrix from $\mathrm{N}$ cells, or by using the associated sum and difference histograms instead of the 2D matrix. In the second approach, less elements have to be filled.

The usual static GLCM features can be computed directly from sum and difference histograms. We have also extracted adaptive low dimensionality GLCM features both from the GLCM matrices and from sum and difference histograms. Tests have been made to estimate the optimum number of gray levels to be used in the analysis of different data sets.

\section{8}

\section{TWO ADAPTIVE TEXTURE FEATURES BASED ON MULTIDIMENSIONAL GRAY LEVEL COOCCURRENCE MATRICES}

Nielsen B. (1,2), Albregtsen F. (1), Danielsen H.E. (2,3)

(1) Department of Informatics, University of Oslo, Norway, (2) Division of Digital Pathology, Norwegian Radium Hospital, Norway, (3) Division of Genomic Medicine, University of Sheffield, England

The Gray Level Cooccurrence Matrix (GLCM) method is a good example of a texture analysis method with a large number of pre-defined features combined with a number of free parameters (number of gray levels, inter-pixel distance, orientation). The method is used to diagnostic and prognostic classification of cell nuclei. We have proposed an approach to low dimensionality adaptive texture feature extraction based on Mahalanobis class distance matrices and class difference matrices. The class distance matrix contains information on which GLCM matrix element that will provide high class discrimination. The class difference matrix contains the difference between the average GLCM of each of two classes. We obtain adaptive features by using squared class distance matrix values as summation weights and the disjoint positive/negative parts of the class difference matrix as the domains of summation in a weighted summation of the GLCM elements.

In the present study we have included several values of the free parameters simultaneously. We then have to work with 3 or 4 dimensional class distance matrices, depending on whether we include several values 
of the inter-pixel distance, or several number of gray levels in the image, or both. We then simply extend the method outlined above to use the squared class distance as weights and the sign of the class difference matrix elements as a bit map in a weighted summation of the 3 or 4D GLCM matrix entries. The number of features extracted from each GLCM matrix remain the same, namely 2 , regardless of the dimensionality of the matrix and the range of the parameters.

\section{9}

\section{AUTOMATED MORPHOMETRIC ANALYSIS OF PERIPHERAL BLOOD SMEARS CELLS IN MICROSCOPIC LARGE FIELDS IMAGES}

Angulo J. (1), Flandrin G. (2), Klossa J. (3)

(1) Center of Mathematical Morphology - School of Mines, Paris, (2) Necker University Hospital, Paris, (3) TRIBVN, Paris, France

Morphological analysis is still essential for diagnosis in haematological disorders. Commercially available automated devices for performing blood film and staining lead to reproducible preparation; a motorised microscope allows to obtain the production of digital microscopic image of large fields (teleslides). We developed an application on lymphoproliferative disorders, erythrocytes morphology and erythrocyte parasites.

An integrated image analysis platform for the segmentation, feature extraction and classification of lymphocytes from peripheral blood teleslides is proposed. In a second phase, the extension of our platform to study the erythrocyte morphology is currently developed. A pre-processing step implements the automated detection of working area of the blood smear which standardises the teleslide production using a routine motorised microscope.

On a teleslide, the leukocyte are detected and the lymphocyte identified and extracted. Each lymphocyte is segmented into two regions: nucleus and cytoplasm. From the nucleus and cytoplasm color images and using mathematical morphology, a set of quantitative parameters are calculated. These measurements yield a first classification of each morphological feature (nuclear size, cell size, N/C ratio, chromatin density, nuclear shape, nucleolus, cytoplasmic basophilia, etc.) into a category (for instance, the nuclear size may be very small, small, medium or large). The set of morphological feature values constitutes the lymphocyte descriptor. The next step involves the use of the morphological descriptor for classifying the lymphocyte into a cellular typology, followed by a classification of the lymphocyte population.

The morphological description provides an objective information which may be indexed into a database together with the teleslide and/or used for (tele)diagnosis procedures and research protocols.

The algorithms have been based on methodical tests from a wide image database. Moreover, at present we are preparing an experience of teleconsensus, entailing the opinion of four experts, in order to validate the descriptors and classification results.

\section{0}

\section{TOWARD AN AUTOMATED TUMOR CLASSIFICATION OF HISTOLOGICAL IMAGES OF NON-SMALL CELL LUNG CARCINOMAS}

Kayser G., Riede U., Kayser K.

Institute of Pathology, University of Freiburg, UICCTPCC, Charite, Berlin, Germany

Aims: To extract information of HE-stained slides of non-small cell lung carcinomas, and to automatically classify the images according to the rules of the WHO. Material and Methods: HE-stained slides of 15 cases of each of the following diagnoses (adenocarcinoma, epidermoid carcinoma, small cell lung carcinoma, large cell carcinoma, healthy lung) have been digitised in a $383 \times 255$ pixel matrix by use of a digital camera $($ Leica DC200) at low $(\times 5)$, moderate $(\times 20)$ and high $(\times 40)$ lens magnification. Feature extraction of the images was performed in terms of gray value analysis $(\times 5)$, texture analysis including Hough transformation $(\times 20)$ and stereologic analysis of nuclei $(\times 40)$. In addition, 5 cases of each of the five diagnostic categories have been automatically classified based upon the data obtained from the teaching set. The segmentation of the images was performed by step-wise thresholding and relational edge-finding of textures lying above the gray value threshold.

Results: Gray value analysis can distinguish all tumor cases from healthy lung tissue as long as more than $20 \%$ of the image area contains tumor cells. No distinction between the different tumor cell types was possible based upon gray value extraction only. Texture analysis can distinguish between small cell lung carcinomas and adenocarcinomas/large cell carcinomas; however not in all cases of epidermoid carcinomas. Stereologic features are useful to separate small cell lung carcinomas from undifferentiated epidermoid carcinomas. 
Discussion: Modern computer technology and image analysing systems can be used as tools for diagnostic assistance in histopathology. Giving predefined classes of diagnoses specific image analysing tools can extract automatically information useful for histopathological diagnosis. In combination with stepwise inclusion of additional diagnoses and screening of complete images it seems likely to create automated diagnosis systems at least for crude diagnoses.

\section{1}

\section{AN AUTOMATED DIAGNOSTIC SYSTEM FOR TUBULAR CARCINOMA OF THE BREAST - AN OVERVIEW OF APPROACH AND CONSIDERATIONS}

Leong J.F.W.-M. (1), Brady J.M. (2), McGee J.O’D. (3)

(1) Nuffield Department of Clinical Laboratory Sciences, John Radcliffe Hospital, University of Oxford, Oxford, United Kingdom, (2) Department of Engineering Science, University of Oxford, United Kingdom, (3) Nuffield Department of Clinical Medicine, John Radcliffe Hospital, University of Oxford, Oxford, United Kingdom

A computer-based automated histopathology recognition system was developed to distinguish benign from malignant lesions. Tubular carcinoma of the breast, which has several reactive and neoplastic mimics, was selected as a model. Archival stained tumour sections from the United Kingdom National External Quality Assurance Scheme for breast pathology and supplementary material from external pathologists formed the study population. A diagnostic process similar to that employed by the histopathologist was adopted, viz, low-power feature extraction and analysis by cluster/glandular groupings followed by high-power confirmation. To circumvent problems of stain variability, greyscale quantisation of images was achieved through Karhunen-Loeve transformation with results suggesting that histological stains provide information primarily through contrast and not colour. Mean nearest neighbour and variance of cell nuclei distances were found to be $100 \%$ effective in distinguishing images which contained diffuse tumour, and no clustering. Gaussian smoothing followed by minimum variance quantisation allowed segmentation of gland clusters. Perona-Malik nonlinear diffusion filter employed prior to intensity thresholding and morphological filtering was $92 \%(7330 / 7973)$ effective in segmenting individual glands. In a set of 62 benign and 52 malignant gland clusters, the features found to discrim- inate tubular carcinoma from benign conditions included $>20 \%$ of glands with sharp-angled edge, cluster area $>150000$ pixels, ratio total gland area: total cluster area $<0.14,>60$ glands per cluster and the ratio average malignant gland area: benign gland area $<0.5$. Suspicious clusters were subjected to high-power feature analysis for nuclear morphology, nucleoli detection and basement membrane assessment. Watershed thresholding achieved nuclear segmentation and nuclear area $>1.3 \times$ mean benign nuclear area was found to have a malignant likelihood ratio of 14.5. Progressive thresholding was used to detect nucleoli. Basement membrane was accentuated by colour segmentation and demonstrated 0.96 sensitivity, 0.89 specificity and 0.92 positive predictive value for distinguishing malignancy.

\section{2 \\ OPTIMAL GRADING OF BREAST CANCER IN DIFFERENT POPULATIONS}

Collan Y. (1), Ikpatt O.F. (1,2), Kronqvist P. (1), Kuopio T. $(1,3)$

(1) Departments of Pathology, University of Turku, SF-20520 Turku, Finland, (2) University of Calabar Teaching Hospital, Calabar, Nigeria, (3) Jyväskylä Central Hospital, Jyväskylä, Finland

Should the principles of grading differ in different populations? This question emerged after the development of the quantitative grading system for breast cancer on a Finnish material of a population in which breast cancer screening is practiced (Kronqvist et al. 1998, 1998, 2000).

In Nigeria breast cancer screening is not practiced, most probably because the economic burden is considered too high among the needs of the national health care. Breast cancers, when diagnosed are far more advanced in Nigeria than in Finland. We evaluated the optimal cutpoints between scores 1, 2, and 3 for morphometric grading in Nigeria (Ikpatt O.F. et al. 2002, 2002) and in Finland. The results are described in table below.

The results show that the patient populations associated with grading scores are different in the different countries if the most powerful distinction of patient groups into survival categories is aimed at. So the answer to the presented question is: yes, the most powerful grading should apply different feature values for grading cutpoints. However, this will mean that also the therapeutic protocols which are associated with grades should be different in the different countries. 


\begin{tabular}{lcc}
\hline Studied feature & Nigeria & Finland \\
\hline $\begin{array}{l}\text { Mitotitic activity } \\
\text { (mitotic figures } / \mathrm{mm}^{2} \text { ) }\end{array}$ & & \\
cutpoint $1 / 2$ & 32 & 17 \\
cutpoint $2 / 3$ & 92 & 32 \\
$\begin{array}{l}\text { Nuclear size } \\
\text { (square mm, i.e., } \mathrm{mm}^{2} \text { ) }\end{array}$ & & \\
cutpoint $1 / 2$ & 47 & 32 \\
cutpoint 2/3 & 111 & 47 \\
$\begin{array}{l}\text { Tubular differentiation } \\
\text { (fraction of fields showing }\end{array}$ & & \\
tubular differentiation, \%) & & \\
The most significant cutpoint & 15 & 59 \\
\hline
\end{tabular}

\section{3}

\section{A NOVEL SPECTRAL MICROSCOPE SYSTEM: APPLICATION IN THE QUANTITATIVE MAPPING OF THE EXPRESSION OF ESTROGEN AND PROGESTERONE RECEPTORS IN BREAST CANCER CELLS}

Papadakis A., Balas C., Stathopoulos E., Berberides K., Nikiforidis G., Delides G.

(1) FORTH-Institute of Electronic Structure and Laser, Heraklion, Crete, Greece, (2) Department of Pathology, Medical School, University of Crete, Heraklion, Crete, Greece

In the present work a novel Spectral Microscope System is presented together with a method for the quantitative assessment of the uptake by histologic samples of stains used in pathology to label tissue features of diagnostic importance. The critical component of the microscope is a Variable Interference Filter-Based monochromator. The system is capable of performing real time spectral imaging in a plurality of spectral bands and micro-spectroscopy in any image pixel, in the spectral range 400-1000 $\mathrm{nm}$. The wavelengthtuning step is $2.4-2.6 \mathrm{~nm}$, while the Full Width Half Maximum in each step is about $1.5 \%$ of the operating central wavelength. The developed system integrates algorithms and calibration procedures for the calculation of the stain-uptake by the tissue. The acquired spectra from both stained tissue and calibration stain solutions enable the calculation of the concentration maps of the stains, even if the latter are multiple and overlap spatially and spectrally. The system was used for the quantitative mapping of the expression of estrogen and progesterone receptors in breast cancer cells. In this particular case, model validation shows that although two stains are employed, capturing of their transmittance at more than ten wavelengths is required in order to obtain an acceptable accuracy. These findings highlight the need for the development and implementation of spectral microscopy in pathology and its potential to introduce novel more reliable diagnostic criteria.

\section{4}

THE AGNORS: STATE OF THE ART

Derenzini M., Montanaro L., Trere D.

Department of Experimental Pathology, Bologna University, Italy

The relevance of the AgNOR parameter in tumour pathology is critically discussed. First, the structural and functional aspects of AgNORs and the biological meaning of their quantitative variations are reviewed to clearly define which indications can be obtained by the use of the AgNOR parameter. The available evidence demonstrates that in continuously proliferating cells the AgNOR parameter indicates the entity of ribosomal biogenesis, which is strictly related to the rapidity of cell proliferation. Therefore, the AgNOR parameter can be considered to represent a marker of cell proliferation rate and, in tumour pathology, should be used only for prognostic purposes. The predictive value of the AgNOR parameter, the only one which indicates the cell growth rate in situ in routinely processed cytohistological samples, is strengthened by combination with kinetics markers indicating the cell growth fraction (i.e., Ki67/MIB1).

\section{5}

RELATIONSHIP BETWEEN C825T POLYMORPHISM OF THE G-BINDING PROTEIN $\beta$-3 SUBUNIT AND PROLIFERATIVE ACTIVITY ASSESSED BY COMBINED AgNOR-FITC METHOD IN COLORECTAL CANCER

Öfner D. (1), Lorenzato M. (2), Siffert W. (3), Ploton D. (4), Schmid K.W. (5)

(1) University Hospital Innsbruck, Department of Surgery, Austria, (2) Laboratoire Pol Bouin, CHU Maison Blanche, University of Reims, France, (3) Institute of Pharmacology, University of Essen, Germany, (4) Unité MéDIAN, CNRS FRE 2141, UFR de Pharmacie, 51096 Reims Cedex, France, (5) Institute of Pathology, University of Essen, Germany 
Introduction: A C825T polymorphism was recently identified in the gene for the G-protein $\beta-3$ subunit. Carriers of the $825 \mathrm{~T}$ allele show an increased risk for hypertension, left ventricular hypertrophy, obesity and enhanced immune response. Moreover, our working group recently found out that carriers of the C825 allele are at 2-fold risk to develop colorectal cancer when compared with carriers of the $825 \mathrm{~T}$ allele. In situ determination of the proliferative activity has become feasible by means of combined AgNOR-FITC method. The aim of the present study was to assess the biological aggressiveness of colorectal tumours with regard to the C825T allele status of the respective patients.

Patients and Methods: DNA was extracted from normal unaffected colon tissue in 122 Patients with colorectal cancer. The C825T polymorphism was determined by PCR and restriction enzyme as recently published. In routinely processed tumour tissues of the respective Patients a double staining technique was carried out, where MIB-1 antibodies were coupled to FITC as recently described. AgNOR area in MIBpositive tumour cells is given in mean $\mu \mathrm{m}^{2}$ per nucleus, additionally the MIB-LI and the Proliferative Index has been calculated.

Results: In 122 Patients with colorectal carcinoma 73 were homozygous C825 (c/c), 45 heterozytegous (c/t), and only 4 Patients homozygous $825 \mathrm{~T}(\mathrm{t} / \mathrm{t})$ allele carriers. The mean $\mathrm{AgNOR}$ area per nucleus ranged from 3.0 to $16.77 \mu \mathrm{m}^{2}$ (mean: 8.4 SD: 2.83 ). MIB-1 LI ranged from 2.15 to 45.6 (mean: 19.4, SD: 7.83) and the PI from 29 to 944 (mean: 338.9, SD: 182.2). Correlation between C825T allele status and AgNOR area, MIB-LI, and PI respectively lacked statistical significance. In uni- and multivariate survival analysis mean AgNOR area per nucleus was found to predict tumour free, but not overall survival.

Conclusion: Proliferative activity of colorectal tumours determined by combined in situ AgNOR-FITC method is independent from C825T polymorphism and predicts independently tumourfree survival of patients.

\section{6}

\section{RELATIONSHIP BETWEEN ANEMIA AND PROLIFERATIVE ACTIVITY ASSESSED BY COMBINED AgNOR-FITC METHOD IN COLORECTAL CANCER}

Steiner P. (1), Lorenzato M. (2), Eichler H. (1), Ploton D. (3), Zitt M. (1), Mittermair R. (1), Öfner D. (1)

(1) University Hospital Innsbruck, Department of Surgery, Austria, (2) Laboratoire Pol Bouin, CHU
Maison Blanche, University of Reims, France, (3) Unité MéDIAN, CNRS FRE 2141, UFR de Pharmacie, 51096 Reims Cedex, France

Introduction: Anemia is a common finding in cancer Patients and was shown to be associated with worse prognosis in a variety of human malignancies, comprising colorectal cancer as published by our workinggroup. In situ determination of the proliferative activity has become feasible by means of combined AgNOR-FITC method. The aim of the present study was to assess the biological aggressiveness of colorectal tumours with regard to the preoperative obtained hemoglobin blood levels of the respective patients.

Patients and Methods: In routinely processed tumour tissues a double staining technique was carried out, where MIB-1 antibodies were coupled to FITC as recently described. AgNOR area in MIB-positive tumour cells is given in mean $\mu \mathrm{m}^{2}$ per nucleus, additionally the MIB-LI and the Proliferative Index (PI $=$ AgNOR area in MIB-positive tumour nuclei $*$ MIB-LI) has been calculated. Hemoglobin blood levels were preoperatively obtained in all Patients. In male anemia is defined as less than $13 \mathrm{~g} / \mathrm{dl}$, in female as less than $12 \mathrm{~g} / \mathrm{dl}$, respectively.

Results: Hemoglobin blood concentrations ranged from 6.8 to $16.5 \mathrm{~g} / \mathrm{dl}$. 45 (41\%) out of 109 Patients with colorectal carcinoma were anemic prior to operation. Mean area of AgNORs in MIB-positive nuclei ranged from 1.6 to $23.4 \mu \mathrm{m}^{2}$ (mean: 8.5 ; SD: 4.08 ), MIB-LI from 4.0 to 45.6 (mean: 19.9; SD: 7.86) and PI from 19 to 512 (mean: 160; SD: 89.7). Overall, tumours of anemic Patients showed higher AgNOR contents and MIB-LI's when compared with tumours of Patients with normal hemoglobin levels prior to operation. But only the PI was statistically significantly associated with the hemoglobin blood level (95\%-CI in anemic Patients ranged from 147 to 215; in Patients without anemia from 129 to $161 ; F=4.3 ; D F=1$; $p<0.05)$.

Conclusion: Colorectal tumours of anemic Patients show enhanced proliferative activity, although only the PI reached statistical significance.

\section{7}

INVASIVE PITUITARY MACROADENOMAS AND NUCLEOLAR ORGANIZER REGION (AgNORs) COUNTS

Szymas J. (1), Hufnagl P. (2), Liebert W. (3)

(1) Department of Pathology, University of Medical Sciences, Poznan, Poland, (2) Institute of Pathology, 
University-Hospital Charite, Berlin, Germany, (3) Department of Neurosurgery, University of Medical Sciences, Poznan, Poland

Tumors of the pituitary gland are usually benign adenomas and account for $10 \%$ of all intracranial neoplasms. However, one-third of pituitary adenomas is invasive at the time of diagnosis. This study was designed to test the hypothesis that AgNOR count is a marker of invasive behavior. Accordingly, we studied two cohorts of pituitary tumors, classified radiologically and intraoperative as invasive or non-invasive. There was a significantly higher partial amount of nuclei with one AgNOR, mean number and corrected mean number of AgNORs per nucleus in invasive tumors $(52.0 \% ; 1.98 \pm 0.90 ; 2.21 \pm 1.26)$ compared to noninvasive adenomas $(32.9 \% ; 1.61 \pm 0.75 ; 1.78 \pm$ 0.94). Early identification of adenomas with likely invasive potential by means of AgNOR count may provide useful information on potential tumor behavior and aid predicting the outcome and designing the treatment to substantially support the light microscopical diagnosis and cell type classification.

\section{8}

\section{THE NUCLEOLAR ACTIVITY CORRELATES WITH p53 AND pRB STATUS IN BREAST CARCINOMAS}

Trerè D., Ceccarelli C., Montanaro L., Santini D., Taffurelli M., Derenzini M.

Department of Experimental Pathology, University of Bologna, Italy

In the present study the relationship between the nucleolar activity and pRB and p53 status has been evaluated in 346 carcinomas of the breast. The nucleolar activity was determined by measuring the AgNOR protein area on routine sections selectively stained by silver-nitrate, while p53 and pRB expression was evaluated by standard immunohistochemistry. Quantitative analysis of all parameters was assessed by image cytometry. The AgNOR areas ranged from 1.63 to $11.63 \mu \mathrm{m}^{2}$, with a mean $( \pm \mathrm{SD})$ value of $4.12( \pm 1.63) \mu \mathrm{m}^{2} . \mathrm{p} 53 \mathrm{im}-$ munostaining ranged from 0 to $99.7 \%$, with a mean $( \pm \mathrm{SD})$ value of $15.77( \pm 29.37) \%$. The mean AgNOR area in the 110 tumors showing p53 accumulation ( $553-\mathrm{LI} \geqslant 5 \%$ ) was significantly greater than in the 236 tumors with p53-LI $<5 \%$ [5.00 \pm 1.66 (SD) $\mu \mathrm{m}^{2}$ vs. $3.71 \pm 1.45$ (SD) $\mu \mathrm{m}^{2}$, respectively; $\left.p<0.001\right]$. The positive correlation between p53 and AgNOR values was maintained also when the two parameters were considered as continuous variables. Twenty three cases showed a negative pRB immunostaining in the cancer cell population ( $\mathrm{pRB}-$ ), and the remaining 323 $(\mathrm{pRB}+)$ had a mean $( \pm \mathrm{SD}) \mathrm{pRB}$ immunostaining of $15.24( \pm 13.89) \%$. The mean AgNOR area of pRBtumors was significantly greater than that of $\mathrm{pRB}+$ tumors $\left[5.59 \pm 1.33\right.$ (SD) $\mu \mathrm{m}^{2}$ vs. $3.99 \pm 1.60$ (SD) $\mu \mathrm{m}^{2}$, respectively; $p<0.001]$. $\mathrm{pRB}+$ and AgNOR values were also related by linear regression analysis, and a positive correlation was found. In conclusion, our results have shown that the nucleolar activity is strictly related with $\mathrm{p} 53$ and $\mathrm{pRB}$ status, providing a morphological confirmation of recent data indicating that the negative control of both p53 and pRB on the cell growth is produced, a part from the block of the G1/S transition, also by the capacity to restrict rDNA transcription.

\section{9}

DIAGNOSTIC VALUE OF NUCLEOLAR ORGANIZER REGIONS (AGNORS) IN BRUSH BIOPSIES OF PRECANCEROUS AND CANCEROUS LESIONS OF THE ORAL

\section{CAVITY}

Boecking A. (1), Remmerbach Th. (2),

Pomjanski N. (1)

(1) Institute of Cytopathology, University of Duesseldorf, Germany, (2) University-Clinics of Leipzig, Germany

Objective: The aim of this retrospective study was to report on the diagnostic accuracy of AgNOR-analysis as an adjunctive diagnostic tool of conventional oral exfoliative cytology taken from suspicious lesions in our clinic and to investigate if this method can contribute to identify progressive lesions out of dysplasias. Study design: Cytological diagnoses obtained from brush biopsies of macroscopically suspicious lesions of the oral mucosa from 75 patients (final diagnoses were: 53 histologically proven squamous cell carcinomas, 11 leukoplakias and other inflammatory oral lesions) and from 11 patients with normal mucosa as a negative control group were compared with histological and/or clinical follow-ups. Five smears were doubtful and seven suspicious for tumor cells in the cytologic report. Number of AgNOR's were countered in 100 squamous epithelial cells per slide after silverrestaining.

Results: Sensitivity of our cytological diagnosis alone on oral smears for the detection of squamous carcinomas was $92.5 \%$, specificity $100 \%$, positive predic- 
tive value was $100 \%$ and negative $84.6 \%$. The best cutoff value of the mean number of AgNOR dots per nucleus distinguishing benign from malignant cells was 4.8. Applying this method to twelve doubtful or suspicious cytological diagnoses we were able to correctly establish the diagnosis of malignancy in ten cases of histologically proven cancers and to reveal benignity in two histologically proven cases. Thus we achieved a positive and negative predictive value of $100 \%$ each.

Conclusions: Smears from brushings of visible oral lesions, if clinically considered as suspicious for cancer, are an easily practicable, cheap, non-invasive, painless, safe and accurate screening method for detection of oral cancerous lesions. We conclude that AgNORanalysis may be a useful adjunct to other methods in routine cytological diagnosis of oral cancer that can help to solve cytologically suspicious or doubtful cases.

\section{0}

\section{THE ROLE OF EXPRESSION OF FAS/FASL ON HEMOPOIETIC PRECURSORS IN THE EVOLUTION OF MYELODYSPLASTIC SYNDROMES}

Lorand-Metze I., Ribeiro E., Lima C.S.P., Metze K.

Departments of Internal Medicine and Pathology, State University of Campinas, Brazil

Increased apoptosis mediated by the Fas/FasL pathway has been considered an important mechanism for the ineffective hemopoiesis observed in myelodysplastic syndromes (MDS). We studied the expression of CD95 and CD95L (Fas/FasL) in CD34 positive cells in bone marrow (BM) of patients with MDS by flow cytometry and correlated it with FAB types, degree of cytopenias, and number of BM blasts. At diagnosis, BM aspirates were made and used for routine morphologic examination, karyotype and flow cytometry. A 3 colour protocol was used: CD45/CD34/CD95 and CD45/CD34/CD95L; 30000 cells were acquired in a FACScalibur equipment. Cell Quest software was used for analysis. We studied 42 cases: 23 RA, 8 RARS, 9 RAEB and 2 CMML. The percentage of blasts showed a correlation with the CD34 positive cells $(r=0.63)$ only in RA/RARS but not in RAEB $(r=0.06)$. The percentage of CD34+ cells expressing CD95 and CD95L showed no correlation with any of the peripheral blood counts, but had an inverse correlation with the percentage of BM blasts $(r=-0.52$ and $r=-0.48$, respectively) and total CD34+ cells $(r=-0.52$ and $r=-0.50$, respectively). In RAEB there was an inverse correlation with CD34+ cells ( $r=-0.67$ and $r=-0.70$, respectively) but not with blasts. The percentage of BM lymphocytes had an inverse correlation with both blasts and CD34+ cells but not with the immature cells expressing CD95 and CD95L. These results favour the hypothesis that an increase in immature cells in MDS is accompanied by a decrease of the ability of these cells to undergo apoptosis. Increased apoptosis seems not to be the only mechanism responsible for the peripheral cytopenias seen in MDS.

\section{1}

\section{CHANGES IN HEMOPOIETIC PRECURSORS} AFTER TREATMENT OF MYELODYSPLASTIC SYNDROMES WITH AMIFOSTINE

Lorand-Metze I., Ribeiro E., Lima C.S.P., Souza C.A., Metze K.

Departments of Internal Medicine and Pathology, Faculty of Medicine, State University of Campinas, Brazil

Amifostine has been used in the treatment of myelodysplastic syndromes (MDS) aiming to reduce the increased apoptosis in bone marrow (BM) precursor cells, and improving peripheral cytopenias. Patients with low risk MDS (RA and RARS by FAB criteria) were treated with amifostine $400 \mathrm{mg} / \mathrm{m}^{2}(3 \times /$ week, 3 weeks, 2 weeks interval). Expression of Faz (CD95) and FasL (CD95L) in CD34+ cells in BM was studied by flow cytometry before and after treatment and correlated with changes in peripheral blood and BM after therapy. We treated 17 patients:11 RA and 6 RARS. After 2 months, 8 patients $(47 \%)$ showed a rise of neutrophil count $>50 \%$. Hemoglobin raised in 1 patient and platelets in 2 patients. According to pretreatment parameters, responders had a significant lower expression of Fas (median 53\%) and FasL (median 17\%) on CD34+ cells than non-responders (median $87 \%$ and $75.5 \%$ respectively). The percentage of BM lymphocytes was significantly lower in responders (median $14.5 \%$ for responders and median $27.4 \%$ for non responders). In responders, the number of CD34+ cells decreased but Fas and FasL did not show significant changes. There was an inverse correlation between change in peripheral neutrophil count after treatment and the percentage of BM lymphocytes before treatment. Therefore, response to amifostine was observed in patients with a lower expression of Fas/FasL in CD34+ cells and a lower percentage of BM lymphocytes. A more severe immunological reaction in BM prevented response to amifostine. 
52

\section{SPECTRAL IMAGING STUDY OF NORMAL LYMPHOCYTES AND LYMPHOBLASTS OF ALL IN CHILDHOOD}

Katzilakis N. (1), Papadakis A. (2), Stiakaki E. (1), Dimitriou H. (1), Balas K. (2), Delides G. (3),

Kalmanti M. (1)

(1) Department of Pediatric Hematology/Oncology University of Crete Medical School, Greece, (2) Institute of Electronic Structure and Laser, Foundation for Research and Technology - Hellas (FORTH), Greece, (3) Department of Pathology, University of Crete Medical School, Greece

Introduction: The morphological distinction of normal lymphocytes from Ll lymphoblasts is in many cases difficult using light microscopy. The distinction and classification is mainly based upon cytochemical features as well as immunologic, cytogenetic and molecular characteristics.

Aim: The quantitative assessment of the spectral absorbance characteristics of the various hemopoietic cell types for the distinction between leukemic cells (and normal ones) since cells with variant biochemical composition uptake in a different way the dye on the routine cytological staining.

Materials and Methods: Spectral analysis has been performed in 30 bone marrow smears following staining with May-Grunwald-Giemsa. 12 children with ALL and 18 children with non-malignant disease aged from 2-14 years old.

The spectral microscope system has been developed at FORTH and is capable of performing imaging of absorbance in a variety of spectral bands, in any image spatial point in a wide spectral range from $400 \mathrm{~nm}$ to $1000 \mathrm{~nm}$.

Results: The analysis showed statistically significant difference $(\rho<0.00005)$ between normal lymphocytes and lymphoblasts based upon the detection, identification and mapping of their spectral absorbance. The wavelengths of $535 \mathrm{~nm}$ and $545 \mathrm{mn}$ corresponded to the maximum absorbance for lymphocytes and lymphoblasts, respectively. The maximum spectral differentiation has been observed at $630 \mathrm{~nm}$ wavelength.

Conclusions: Spectral analysis of bone marrow smears from children with Acute Lymphoblastic Leukemia has shown that imaging microspectroscopy could provide an additional quantitative index correlated with the biochemical, functional and structural status of the cell. This could contribute in the introduction of more objective and reliable diagnostic criteria in the diagnosis and classification of acute leukemia.

\section{3}

\section{DIAGNOSTIC DISCREPANCY BETWEEN MORPHOLOGY/CYTOCHEMISTRY AND IMMUNOPHENOTYPING IN 420 NEW CASES OF ACUTE LEUKEMIA}

Azordegan N., Kowsary F., Sotoudeh M., Nikoogoftar M.

Sina and Baghiyatallah hospital, Tehran, Iran

Background: Morphological examination of bone marrow smears with cytochemical staining forms the basis for French American British (FAB) classification. However immunophenotyping by multiparameter flowcytometry has become an invaluable tool in diagnosis, classification and monitoring of patients with acute leukemia.

Objective: This study aims to compare the results of FAB classification with flowcytometric immunophenotyping in diagnosis of acute leukemia and stress on the value of immunophenotyping since many distinct types are known to warrant specific therapy and carry predictable prognoses.

Methods: Bone marrow aspirates from 420 cases of acute leukemia were analyzed using morphology and cytochemical staining characteristics. Immunophenotyping of all cases were also determined by flow cytometry using monoclonal antibodies. Results compared.

Results: Classification of 420 cases of acute leukemia as assessed by flowcytometry are as follows: 280 $(66.7 \%)$ acute lymphoblastic, 135 (32.1\%) acute myeloid, $3(0.7 \%)$ biphenotypic, $2(0.5 \%)$ unclassified cases.

Of 280 cases designated lymphoid origin by flowcytometry, 257 (91\%) were diagnosed as such by morphology and cytochemistry.

The discordant cases were diagnosed as myeloid (23 cases).

Of 135 immunophenotypically proved acute myeloid leukemia, $115(85 \%)$ were diagnosed as myeloid by morphology and cytochemistry. The cases with discordant diagnosis were identified as lymphoid leukemias (20 cases).

Immunophenotypically diagnosed cases of biphenotypic (3 cases) and unclassified ( 2 cases) leukemia were diagnosed as lymphoid leukemia by morphology/ cytochemistry. 
Conclusion: By comparing the results obtained by morphology/cytochemistry according to FAB classification with flowcytometry, this study stresses the importance of examining the bone marrow aspirates by flowcytometry for definite diagnoses and selection of appropriate treatment protocols.

54

\section{DNA PLOIDY DISTRIBUTION IN 49 CASES OF GERM CELL TUMORS OF THE OVARY}

Kildal W. (1), Kærn J. (2), Abeler V. (1), Sudbø J. (3), Danielsen H.E. $(1,4)$

(1) Departments of Pathology, (2) Gynecologic Oncology, (3) Medical Oncology and Radiotherapy, The University Clinic of The Norwegian Radium Hospital, 0310 Oslo, Norway, (4) Division of genomic medicine, University of Sheffield, Sheffield, United Kingdom

Malignant germ cell tumors of the ovary (mOGCT) have been difficult to study due to their infrequency, accounting for less than $5 \%$ of all ovarian malignancies. Measuring DNA content with high-resolution image cytometry is feasible, and we have recently demonstrated prognostic value in human malignancies and premalignancies. The information of DNA ploidy in mOGCTs is limited. To reveal if our highresolution DNA ploidy system could add information on DNA content, we performed analysis on 49 mOGCT.

Forty-nine cases of mOGCT, of which 20 tumors were classified as dysgerminoma (DG), 17 as immature teratoma (IT), 10 as endodermal sinus tumor (EST), 1 as embryonal carcinoma (EC) and 1 as choriocarcinoma (CC) were studied. Eight of these lesions also contained another tumor component, which was not possible to evaluate separately. Image cytometry were performed to discern the DNA ploidy distribution in this material.

Fifteen of the 49 tumors (33\%) were diploid, 14 (31\%) were tetraploid, $2(4 \%)$ were polyploid and $14(31 \%)$ were aneuploid. Five (11\%) of the 47 patients studied, died of disease during the follow up. None of these were diploid. One was tetraploid, 1 was polyploid and 3 were aneuploid. All the ITs were diploid, while for DGs and ESTs $47 \%$ and $50 \%$ were tetraploid and $53 \%$ and $50 \%$ were aneuploid/polyploid, respectively. All patients with FIGO stage I disease, diploid DNA ploidy distribution, pure DG, IT and CC survived the disease for at least 44 months of follow up.

\section{5}

\section{DNA PLOIDY AND CHROMOSOMAL COPY NUMBER ANALYSIS OF BILATERAL FEMALE}

Kildal W. (1), Kraggerud S.M. (3), Abeler V.M. (1), Heim S. (3), Tropé C.G. (2), Kristensen G.B. (2), Risberg B. (1), Lothe R.A. (3), Danielsen H.E. (1,4)

(1) Departments of Pathology and (2) Gynecologic Oncology, (3) Department of Genetics Institute for cancer research, The University Clinic of The Norwegian Radium Hospital, 0310 Oslo, Norway, (4) Division of genomic medicine, University of Sheffield, Sheffield, United Kingdom

We present a case report of a 16-year-old, phenotypic female with bilateral dysgerminomas and unilateral gonadoblastoma. In contrast to the common observations, this dysgerminoma metastasized to the peritoneal cavity. The constitutional karyotype for the patient was $46 \mathrm{XY}$ and the DNA ploidy classifications of the dysgerminomas were tetraploid in the right and aneuploid in the left ovary. The gonadoblastoma component was tetraploid, while the metastasis showed an aneuploid DNA ploidy distribution. The chromosomal copy number examined by $\mathrm{CGH}$ showed 3 gains in the dysgerminoma of the right and 6 gains in the left ovary. Two gains and one loss were observed in the gonadoblastoma component. The metastasis showed comparable DNA ploidy pattern and chromosomal copy number changes as the dysgerminoma of the right ovary. In conclusion, the metastasis is most likely from the dysgerminoma of the right ovary. Possibly, the specific genetic damage in the primary tumor may be related to its ability to progress.

\section{6}

\section{DNA CLEARING. SEARCH FOR PROTEINS} INVOLVED

Levina V.V. (1,2), Drobchenko E.A. (1),

Shabalina E.V. (1), Sukhareva E.B. (1), Guzova I.V. (2),

Govorun V.M. (3), Goufman E.I. (3),

Serebryakova M.V. (3), Tikhonova O.V. (3),

Khryapova E.V. (3), Toropyguine I.Yu. (3),

Maksimov B.I. (3), Lokhov P.G. (3),

Moshkovskii S.A. (3), Archakov A.I. (3), Kytola S. (4)

(1) Petersburg Nuclear Physics Institute RAS, Russia,

(2) Institute of Cytology SPb, RAS, Russia, (3) Institute of Biomedical Chemistry RAMS, Moscow, Russia, (4) Institute of Medical Technology University of Tampere, Finland 
Novel mechanism affecting the drug resistance in tumour cells: active dissociation of noncovalently bound agents from DNA or "DNA clearing" is the subject of this work. Flow cytometry technique and fluorescent dye Hoechst 33342 is used to study the process of "DNA clearing" in living cells.

The process of "DNA clearing" is energy-dependent and could be suppressed by topoisomerase- 2 inhibitors, protein kinase inhibitors and DNA breaks.

The method of stepwise selection with increasing concentrations of Hoechst 33342 was used to develop a set of Hoechst 33342 resistant rodent and human cell lines with enhanced level of "DNA clearing". Selected cell lines showed cross-resistance to various DNA-damaging chemicals: mitomycin C, ethidium bromide, camptothecin, and etoposide and resistance to high temperature treatment when compared with parent MCF7 one. The overexpression of constitutive and heat induced major stress protein Hsp70 was detected in theMCF7HoeR-7 cells.

To reveal the possible chromosome elements responsible for DNA clearing and proteins involved in the process we analyse parental MCF7 and independently selected multidrug resistant MCF7HoeR-3, MCF7HoeR-7 and MCF7HoeR-8 lines on different steps of selection. Comparative Genomic Hybridisation (CGH) and Spectral Karyotyping (SKY) methods are used to analyse chromosome alterations and the conventional 2D-EF technique - to study proteomes differences. A comparison of 2D gels shows changes at 17 protein spots, being more expressed in strongly resistant mutant. Several differential proteins were identified by MALDI-MS.

\section{7}

\section{THE ROLE OF GLARE AND DIFFRACTION CORRECTIONS IN ESTIMATING LYMPHOCYTE IOD VALUES IN DNA CYTOMETRY}

Clinch N.F., Maddison J., Danielsen H.E.

Department of Pathology, Norwegian Radium Hospital, Oslo, Norway and Fairfield Imaging Ltd., Tunbridge Wells, Kent, United Kingdom

Measures of the integrated optical density (IOD) of Fuelgen-stained cell nuclei in conventional imagebased DNA cytometry do not translate quantitatively into nuclear DNA content. For this reason the interpretation of DNA Ploidy histograms is based on locating the correct 2c position in the IOD axis. The histogram is then evaluated in the light of current understand- ing of the normal cell-cycle, and it is commonplace to use the presence of (diploid) lymphocytes within a tissue sample to provide "internal control nuclei" that can give a direct indication of the IOD corresponding to 2c. However there is a well-known difficulty with this approach; lymphocyte chromatin is highly condensed and unless suitable corrections are applied for diffraction effects at the nuclear edge, and for the presence of stray light within the microscope ("glare"), the measured IOD for these very small nuclei can significantly under-estimate their "true" diploid DNA content relative to that in, say, diploid epithelial or stromal cell nuclei. Fortunately there are well-known methods for applying these corrections to the raw image data, but still it is worthwhile to look for methods that do not rely on the use of arbitrary constants in the IOD correction process. Here we report a new method for diffraction-correction, and we examine the validity of the concept of "glare" as a uniform, distributed light intensity to be subtracted from every pixel in the digital image. A potentially significant contributor to error in lymphocyte IOD measurement is the presence of symmetrical fluctuations in measured object transmittance due to CCD camera noise. Such transmittance fluctuations can significantly alter the measured mean OD of an object in the microscope.

\section{8}

\section{CHROMOSOMAL INSTABILITY IN FLAT ADENOMAS AND CARCINOMAS OF THE COLON}

Postma C. (1), Hermsen M.A.J.A. (1),

Baak J.P.A. (1), Mueller J.D. (2), Mueller E. (3), Bethke B. (4), Stolte M. (4), Meijer G.A. (1)

(1) Institute of Pathology, VU University medical center, Amsterdam, The Netherlands, (2) Department of Surgery and (3) Institute of Pathology, Klinikum rechts der Isar, Technische Universität München, Munich, Germany, (4) Institute of Pathology, Klinikum Bayreuth, Bayreuth, Germany

Controversy exist on the pathogenesis of colorectal carcinomas. While according to the classic adenomacarcinoma sequence all carcinomas arise from adenomatous polyps, recent observations have demonstrated the existence of a flat adenoma to carcinoma pathway. We recently have demonstrated in classic polypoid colorectal adenomas and carcinomas the existence of subsets of tumours with different patterns of chromosomal instability (CIN). 
Aim of the present study was to compare the patterns of CIN in a series of flat adenomas with high-grade dysplasia as well as early flat carcinomas by comparative genomic hybridisation ( $\mathrm{CGH}$ ) to those previously found in polypoid adenomas and carcinomas with similar degrees of neoplasia.

Formalin-fixed paraffin embedded tissue samples of 6 flat adenomas and 12 flat carcinomas of the colon were analysed by CGH. Patterns of chromosomal aberrations were analysed by hierarchical cluster analysis.

The most frequent alterations in the flat adenomas were loss of $17 \mathrm{p}(50 \%), 18 \mathrm{p}(50 \%)$ and $18 \mathrm{q}(83 \%)$, and gain of $20 \mathrm{p}(50 \%)$ and $20 \mathrm{q}(83 \%)$. In the flat carcinomas loss of $4 \mathrm{p}(42 \%), 4 \mathrm{q}(58 \%)$ and $18 \mathrm{q}(92 \%)$, and gain of $7 \mathrm{p}(42 \%), 7 \mathrm{q}(42 \%), 17 \mathrm{q}(42 \%), 20 \mathrm{p}(58 \%)$ and $20 \mathrm{q}$ $(92 \%)$ occurred most frequently. Three cases showed high-level amplifications on 20q13.

Hierarchical cluster analysis of combined flat and polypoid tumours resulted in two clusters. While the polypoid lesions occurred in both clusters, the vast majority of flat adenomas (5/6) and flat carcinomas (11/12) segregated into a single cluster (together with part of the polypoid lesions). This cluster was mainly marked by $18 \mathrm{q}$ loss $(66 \%)$ and $20 \mathrm{q}$ gain $(59 \%)$.

These results suggest flat adenomas and carcinomas of the large intestine are genetically more homogeneous than their polypoid counterparts.

\section{9}

\section{PROGNOSTIC SIGNIFICANCE OF DNA-IMAGE-CYTOMETRY IN GASTROINTESTINAL AND LUNG CARCINOIDS}

Böcking A. (1), Raatz H. (2), Hauptmann St. (2)

(1) Institute of Cytopathology, University of Düsseldorf, Germany, (2) Institute of Pathology, Humboldt University of Berlin, Charité, Germany

Aims: To evaluate and compare the prognostic validity of various clinical, histopathological, immunohistochemical and DNA-cytometric variables in patients with carcinoid tumors of the gastrointestinal tract and lung.

Methods: Resected carcinoids in 44 patients were investigated concerning the correlation of tumor size, histological type (A-E) and differentiation, localization, infiltrative growth, residual tumor, immunohistochemisty (nine different antibodies) and DNA-imagecytometry (ten different indices) with the occurrence of local recurrence, lymphnode or distant metastases and survival time of the patients. Follow up was up to 108 months.

Results: Histological type, infiltrative growth, occurrence of S-100 or pancreatic polypeptide positive cells, DNA-histogram type, 5c Exceeding events (5cEE) and the $2 \mathrm{c}$ Deviation Index (2c DI) showed univariate prognostic relevance. In multivariate regression analysis (Cox model) $5 \mathrm{cEE}>0$ revealed to be the prognostically most relevant parameter before histological type and other variables.

Conclusion: DNA-image-cytometry, which can be performed preoperatively on fine needle aspiration biopsies of carcinoids allows the prediction of local recurrence, lymphnode- and distant metastases and survival time after operation in patients with carcinoid tumors.

\section{0}

\section{GENOMIC PROFILING OF GASTRIC CANCER PREDICTS LYMPH NODE STATUS AND SURVIVAL}

Weiss M.M. (1), Kuipers E.J. (2), Postma C. (3), Snijders A.M. (4), Siccama I. (5), Pinkel D. (4), Westerga J. (6), Meuwissen S.G.M. (1), Albertson D. (4), Meijer G.A. (3)

(1) Departments of Gastroenterology and (3) Pathology, VU University Medical Center, Amsterdam, The Netherlands, (2) Department of Gastroenterology and Hepatology, Erasmus University Medical Center, Rotterdam, The Netherlands, (4) UCSF Cancer Center, San Francisco, California, USA, (5) KIQ Ltd., Amsterdam, The Netherlands, (6) Department of Pathology, Slotervaart Hospital, Amsterdam, The Netherlands

Introduction: Pathogenesis of gastric cancer is driven by an accumulation of genetic changes that to a large extend occur at the chromosomal level. Therefore, the patterns of chromosomal instability in gastric carcinomas and their clinical correlations were analyzed by microarray based comparative genomic hybridization (microarray $\mathrm{CGH}$ ). With this technique, genomewide chromosomal copy number changes can be studied with high resolution and sensitivity.

Material and Methods: Microarray CGH was applied to a series of 35 gastric adenocarcinomas. using a genome-wide scanning array with 2214 BAC and P1 clones spotted in triplicate. Each clone contains at least one STS for linkage to the sequence of the human genome. These arrays provide an average resolution of $1.4 \mathrm{Mb}$ across the genome. Results of hierarchical cluster analysis of the normalized $\log _{2}$ tumor to normal flu- 
orescence ratios of all clones and tumors were correlated to clinicopathological data including survival.

Results: Hierarchical cluster analysis revealed three different genomic gastric cancer profiles (cluster 1, 2, and 3), with cluster 2 showing a pattern intermediate between cluster 1 and 3. These profiles were significantly correlated with lymph node status $(p=0.02)$. Moreover, gastric cancer cases from cluster $3(n=14)$ showed a significantly better prognosis than those from cluster $1(n=9)$ and $2(n=6),(p=0.02)$.

Conclusions: Genomic profiling of gastric adenocarcinomas based on microarray analysis of chromosomal copy number changes predicts lymph node status and allows for discriminating a subgroup of patients with high risk of lymph node metastasis. This latter observation could clinically be highly relevant for selecting candidate patients for extended lymph node resection.

\section{1}

\section{PATTERN OF DISTRIBUTION AND PROGNOSTIC VALUE OF ANGIOGENESIS IN PANCREATIC DUCT CARCINOMA: A SEMIQUANTITATIVE IMMUNOHISTOCHEMICAL STUDY OF 45 PATIENTS}

Linder S., Blasjo M., von Rosen A., Parrado C., Falkmer U.G., Falkmer S.

Department of Surgery, Sodersjukhuset, Karolinska Hospital, Faculty of Medicine, Karolinska Institute, Stockholm, Sweden

In specimens obtained from resected pancreata, the intratumoral microvessel density (IMD), the poliferation rate of the neoplastic parenchymal cells, and their p53 protein expression were assessed. The sources of errors were great in the measurements of the IMD. This statement can be illustrated by the finding that when the IMD was calculated by manual counting in five areas of intense neovascularization (hot spot regions), using $\times 200$ and $\times 400$ magnifications, the numbers of microvessels per square millimeter were $65 \pm 23$ and $106 \pm 8$, respectively, which reflects a significant difference. Two patterns of microvessel distribution could be identified: one with hot spots only in the stroma ( $n=19$ ) and one in which the hot spots were located in areas of neoplastic parenchyma (including its stroma) ( $n=26)$. The IMD was significantly greater in the latter group.

There was no general correlation of neoplastic disease with the IMD. However, when a scoring system was used to assess the angiogenesis, hot spots in areas of neoplastic parenchyma were associated with a greater proliferation rate of the tumor cells, and with a short length of survival of the patients from their neoplastic disease.

\section{2}

DMD (DIGITAL MICROMIRROR DEVICE) ENABLED MICROSCOPY: TAKING THE MICROSCOPE IN NEW DIRECTIONS

MacAulay C., Lane P., Dlugan A., Lam S.

Cancer Imaging, B.C. Cancer Agency, USA

Placing computer controlled spatial light modulators (DMD) in the light path of a microscope enables a variety of simple novel adaptations.

Conventional endoscopy is limited to imaging macroscopic views of tissue. DMDs make it possible to shoehorn a confocal microscope down a coherent fibre optic imaging bundle enabling confocal imaging of cells and tissue structure in vivo through the biopsy channel of an endoscope The feasibility of sub-micron, (pseudo_real-time) imaging of individual cells and accompanying tissue architecture in the epithelial lining of an organ or organ system using both reflectance and tissue auto-fluorescence modes of imaging is possible. Currently, multiwavelength reflectance confocal microendoscopy with $\sim 1 \mu \mathrm{m} x-y$ resolution and $7 \mu \mathrm{m}$ $z$ resolution has been achieved.

Most CCD based microarray fluorescence readers suffer from having $\sim 10^{3}$ dynamic range (12-14 bitdigitization). This dynamic range can be improved using an appropriate coordinate mapping function applied to a DMD in the illumination path of the microscope to direct known amounts of excitation light at specific cDNA spots, such that darker spots receive more excitation light increasing their measured brightness and bright spots receive less light so they do not saturate the CCD. In this fashion, the dynamic range of a DMD-CCD microarray reader system becomes essentially that of the DMD plus that of the CCD (2022 bits).

For a microscope with the DMD in a conjugate image plane of the back focal plane of the objective, optical tomography reconstruction of absorption stained sections and cytology is possible on a microscopic scale through the use of filtered Back-Propagation algorithms allowing 3D reconstruction images.

These and other applications of spatial light modulators to microscopy will be discussed. 
63

\section{PRACTICAL APPLICATION OF GAUSSIAN BLURRING TO DETERMINE AND ADAPTIVELY CORRECT UNEVEN ILLUMINATION (VIGNETTING) IN DIGITAL MICROSCOPY IMAGES}

Leong J.F.W.-M. (1), Brady J.M. (2),

McGee J.O’D. (3)

(1) Nuffield Department of Clinical Laboratory Sciences, John Radcliffe Hospital, University of Oxford, Oxford, United Kingdom, (2) Department of Engineering Science, University of Oxford, United Kingdom, (3) Nuffield Department of Clinical Medicine, John Radcliffe Hospital, University of Oxford, Oxford, United Kingdom

Many digital microscopy images suffer from poor illumination at the periphery (vignetting) often attributable to factors related to the light path between the camera and the microscope. A commonly-used method to date has been to use the illumination of an empty field as a correction filter (white shading correction). We outline an alternative retrospective shading correction method that does not require this additional step and is based upon the intrinsic properties of the image which are revealed through Gaussian smoothing. The technique can be implemented within Adobe Photoshop or other graphics editing software and provides an effective means of optimising digital microscopy image appearances for printing, image analysis or telepathology.

\section{4}

\section{OPTIMISED GREYSCALE QUANTISATION} THROUGH PRINCIPAL COMPONENT ANALYSIS AS A MEANS OF COMPENSATING FOR HISTOCHEMICAL STAIN VARIATION

Leong J.F.W.-M. (1), Brady J.M. (2), McGee J.O’D. (3)

(1) Nuffield Department of Clinical Laboratory Sciences, John Radcliffe Hospital, University of Oxford, Oxford, United Kingdom, (2) Department of Engineering Science, University of Oxford, United Kingdom, (3) Nuffield Department of Clinical Medicine, John Radcliffe Hospital, University of Oxford, Oxford, United Kingdom

The proliferation of digital imaging devices for microscopy has facilitated microscopy image capture for the purpose of presentation of publication. While such images are frequently captured in 24-bit (true color), for economic reasons, print journals still prefer blackand-white images. Greyscale ("black-and-white") images also play a significant role as an intermediate phase in image processing and analysis.

If 24-bit colour is visualised as a three-dimensional cube, with each axis corresponding to red, green or blue, then a greyscale image can be derived by defining a line through this cube. Many pathologists do not realise that an infinite number of possibilities exist, each favouring particular color characteristics of the original image.

We investigated the common methods of greyscale quantisation from 24-bit colour hematoxylin and eosin stained histological images and compared these methods with principle component analysis. A series of breast lesions at low and high power were used, with the results compared visually with the original images. Using the first principal component derived from the colour image provided the most satisfactory greyscale image with both stromal and glandular features preserved. We conclude that this is a useful technique that ensures a consistent greyscale image independent of histochemical stain variation. Additionally, the possibility is raised that that histochemical stains provide information through contrast, rather than colour.

\section{5}

\section{CONCEPT AND REALISATION OF DIGITAL MICROSCOPY IN HUNGARY}

Gombás P. (1), Molnár B. (2)

(1) MI Central Hospital, Department of Pathology, Hungary, (2) Digital Microscopy Laboratory, 2nd Department of Medicine, Semmelweis University, Budapest, Hungary

AIMS: Although telepathology techniques have been accepted as global method there are serious doubts to implement cyber age tools in professional practice. Static telepathology has proven biased due to the low explicit/implicit ratio of transmitted information, dynamic telepathology can not be automated using remote controlled conventional microscopes. Our aim was to elaborate a system which minimise drawbacks of traditional telepathology methods and enables new functions in routine histopathology.

METHODS: Digital images of gastrointestinal histopathological samples were captured with a bright field transmitted light microscope and in house developed slide scanner. Images of selected biopsies have been joined to digitised slide using different scanning meth- 
ods. Digitised slides has been stored, transferred to remote locations and analysed using various morphometric algorithms.

RESULTS: The value of bias of transferred digital information can be reduced to zero digitising the entire graphic information of histopathology specimen. We tested the following systems:

1. Manual digitising of the specimen via $x$ and $y$ axes using RGB microscopic camera and frame grabber is extremely laborious and inappropriate in the routine practice.

2. Automatic scanning of slides applying traditional microscope, which is equipped with motorised stage digitises the entirety of selected slides but not able for fully automated capturing of slide series.

3. Histological slide scanner without conventional microscope, which contains software controlled stage and laser based auto-focus, enable fast and completely automated construction of digitised slides. A series of conventional and digital biopsy slides has been evaluated and compared, which has not presented diagnostic differences.

CONCLUSION: Basic question of digitised slide's application in the routine practice is how the specimen has been captured. Application of digital slide captured by slide scanner dissolves bias in telepathology practice and enables a new agenda for expert discussions, consultations, archiving, education and quality assurance.

\section{6}

\section{METHODOLOGICAL ASPECTS OF INTERPHASE FISH IN DIAGNOSTICS}

Friedrich K. (1), Meyer W. (1), Haupt I. (1), Stief K. (1), Hilt A. (1), Haroske A. (2),

Kunze K.D. (1), Baretton G. (1)

(1) Institutes of Pathology of University of Technology Dresden, Germany, (2) General Hospital DresdenFriedrichstadt, Germany

Interphase FISH is becoming an integral part of diagnostics, prognostics and disease monitoring. The use of archived tissue reveals information about changes in the genome, which may be valuable for therapeutic decisions.

Hitherto, systematic studies of methodological aspects like the kind of samples, different indications as well as different probes and the reproducibility are still rare. Thus, the study was aimed to analyze the influence of different kinds of samples and probes as well as the reproducibility in interphase FISH on routinely processed tissue.

Imprints of unfixed tumor tissue, paraffin section with different section thickness $(2,4,6 \mu \mathrm{m})$ and disintegrated $50 \mu \mathrm{m}$ paraffin sections are hybridized with probes for amplification (HER-2neu), deletion (p53) and translocation (bcr-abl). One hundred nuclei were counted per slide. The reproducibility of interphase FISH was tested by ten consecutive different hybridizations of the HER-2neu probe on disintegrated $50 \mu \mathrm{m}$ sections of the same paraffin sample. These hybridizations were evaluated by two independent observers.

The study of inter- and intraobserver variability detected sampling errors. The observation of hybridization over the time showed a sufficient quality of hybridization for the detection of HER-2neu amplification with the threshold of $10 \%$ of counted nuclei with at least four HER-2neu signals and a HER2 neu/centromer 17 ratio of two.

The imprints and the disintegrated paraffin sections avoid the problems of artifacts by cutting nuclei and overlapping nuclei. On the other hand, the histomorphology is not completely conserved and small foci of interest are not yet detectable.

The study stress the necessity of quality control by suitable controls as well as standardized thresholds depending on used samples, on the probes and the indication of interphase FISH.

\section{7}

\section{WEB BASED TISSUE MICROARRAY MANAGEMENT SYSTEM}

Demichelis F. (1), Barbareschi M. (2), Dalla Palma P. (2), Dell' Anna R. (1)

(1) Medical Informatics and Telemedicine Laboratory, ITC/irst, Trento, Italy, (2) Inst. of Pathology, S. Chiara City Hospital, Trento, Italy

Enormous data sets and information are involved in TMA based studies; well structured data management system are mandatory. The incremental need to share data among different institutions and multicentric studies suggests the implementation of web based systems and the definition of a standard to be applied in exchanging data (see http://www.pathinfo.com/jjb /tmadtd2.htm). Data concerning with TMA based studies are biological/genetic, clinical and morphologic. Digital images of entire array slides and of single core 
sections are necessary for eventually automated scoring and for exhaustive storage.

Relational database addresses the requirements to organize in an efficient manner all the data. In our system insertion, updating and retrieval of data are provided through web pages (Active Server Pages) constructed to satisfy different data entry types and peculiar work phases. Different views are designed to map the workflows of different work phases.

The intrinsic difficulty of TMA samples analysis by conventional microscope (hundreds of tumours spots on the same slide) suggests the storage of digital images of single core section, uniquely related to the tumour identifier, and of overview images of entire slides.

Robotic microscopy is well suitable for this kind of issues. Digital images of core sections enable the pathologists to easily compare the immunostainings tumour samples on computer screen through a microscope simulator. Having a TMA management system the problem shifts from data generation to data-mining and deriving information from such material (Journal of Pathology 195 (2001), 1-2). Patterns in TMA biological data of gene expression have to be evaluated. Correlations of expression with clinical and follow up data are of main interest for prognosis and therapeutic investigation. To assess the screening approach to the analysis of potential markers, classical statistics as uni-multivariate analysis together with supervised and unsupervised cluster analysis will be utilized.

This work has been granted by the Ministry of Public Health of Italian Government.

\section{8}

\section{HIGH THROUGHPUT ANALYSIS OF HER-2NEU IN BREAST CANCER BY FISH AND IMMUNOHISTOCHEMISTRY ON MULTI TISSUE ARRAYS}

Friedrich K., Stief K., Haupt I., Klenk U., Meyer W., Kunze K.D., Baretton G.

Institute of Pathology, University of Technology Dresden, Germany

Out of the background of the therapeutic possibilities of trastzumab, the HER-2neu has emerged. The role of HER-2neu protein overexpression or gene amplification in prognostication of breast cancer is controversially discussed. One reason for this controversy is the lack of standardized studies with a high number of cases with long time follow up.
The technique of multi tissue array may overcome this problem by analyze of up to 500 tumor samples by the same preparation protocol.

The study was aimed to analyze the value of multi tissue arrays for FISH and immunohistochemistry in breast cancer, the correlation between changes of HER-2neu gene and protein expression and the prognostic impact of changes of HER-2neu.

At least two samples of each of 600 breast cancers with known follow up were selected for multi tissue arrays. The samples were arranged at six multi tissue arrays. Each array includes 30 samples of nonneoplastic breast tissue as normal controls. The FISH analysis was performed by PathVysion HER-2 (Abbott), the immunohistochemical detection of HER2neu was done by a polyclonal antibody (DAKO; antibody A0485).

More than $90 \%$ of arrays showed a sufficient result of hybridization and immunohistochemistry. More than $90 \%$ of arrays allow the counting of at least hundred nuclei. Only few differences were detected between different arrays of the same tumor, especially for amplifications. The amplifications of HER-2neu correlated with a strong positive reaction in the immunohistochemistry.

In summary, the use of multi tissue arrays allows standardized analyses of changes at gene (especially of gene amplifications) as well as at protein level in a high number of cases. Thus follow up studies of subgroups of tumors with specific clinicopathological features may be possible.

\section{9}

DIGITAL QUALITY CONTROL IN CYTOLOGY: IDEAS AND METHODS

Beltrami C.A. (1), Demichelis F. (2), Della Mea V. (3), Dalla Palma P. (4), Forti S. (2)

(1) Inst. of Pathology, University of Udine, Italy, (2) Medical Informatics and Telemedicine Laboratory, ITC/irst, Trento, Italy, (3) Department of Mathematics and Computer Science, University of Udine, Italy, (4) Inst. of Pathology, S. Chiara City Hospital, Trento, Italy

The uniqueness of the glass slide is a crucial problem in the implementation of quality control schemes in cytology, because it makes difficult to share specimens as needed.

Among the various quality control methods, proficiency testing is a significant component, and is meant 
at evaluating the diagnostic skills of interested subjects. Proficiency testing is also particularly crucial in cytologic screening.

The availability of modern technologies allows us now to digitize and store full glass slides, in the form of thousands of images organized in matrices. This way, the traditional glass support for specimens may be transformed into a digital one, which brings all the typical advantages of digitals objects, including duplicability. Although not easy to store and manage, digital glass slides can be the right way towards the real implementation of quality control programmes, by distributing digital copies of specimens to all participants.

The present work will describe the digital slide features and constraints, together with a possible scheme for digital quality control.

70

\section{QUALITY REQUIREMENTS AND CONTROL} OF IMAGES IN TELEPATHOLOGY

Schwarzmann P.

University of Stuttgart

Quality control and documentation of images in pathology has become an issue with the advent of Telepathology, because in this situation the person selecting and acquiring image material is different from the person evaluating the image material.

The receiver has to rely fully on the image material he gets with respect to

- selection of adequate fields of view for diagnosis,

- resolution and field of view, contrast, color and brightness,

- correct image acquisition, transmission and presentation.

In today's telepathology systems tradeoffs have to be made between resolution and field of view, online impression and data rate. These tradeoffs have to be established carefully dependent on the application, the requirements of the pathologists and the economic efficiency of a system. Results of fieldtests and longer routine applications provide decision arguments for effective system control.

An efficient tool to maintain quality of a telepathology service is the permanent control and documentation of parameters of image acquisition, transmission and display of the image data. Criteria have to be derived in accordance with the importance of an image feature for the diagnostic quality. This means, transmitted images have to preserve diagnostic cues. These criteria are on one side the subjective visual impression of the image and on the other side objective criteria like the visibility of geometric details, color details and hue of staining, quantitative extinction and last but not least the conservation of the familiar impression in conventional microscopy.

The need for quality control and documentation becomes still more relevant if remote computerized image evaluation is also considered.

The control, documentation and monitoring of image quality within telepathology sessions is still neglected in most applications due to missing reported experiences and demonstration of systems including such facilities.

\section{1}

AN RDF-BASED FRAMEWORK FOR DIGITAL PATHOLOGY CASES

Della Mea V. (1), Brauchli K. (2), Demichelis F. (3)

(1) Department of Mathematics and Computer Science, University of Udine, Italy, (2) Institute of Pathology, University of Basel, Switzerland, (3) Laboratory of Medical Informatics and Telemedicine, ITC/irst, Trento, Italy

In a telepathology process, the case is centered around images, but includes some data and features depending on the case source (i.e., electronic medical record, glass slide) and author, some others determined by the image technology used for its delivery, and finally some more information, which highly depends on the specific aim that caused the case creation and delivery. We may distinguish among basic case information, image information (including technical details), aimoriented information.

Such information may be represented by metadata associated with the case, where the case is in turn a set of images (just still images, or digital slides, etc.) plus clinical history, plus author and indexing information. In the present evolution of the Web towards the Semantic Web, metadata can be represented by means of RDF (Resource Description Framework), which provides for semantic constructs suitable for metadata, in a XML syntax. Furthermore, part of the metadata needed for describing a resource (non only online) are already standardized under the Dublin Core initiative (i.e., Creator, title, Date, etc.).

As a preliminary step, we developed a generic model for digital pathology cases, including the concepts of image, plane and stack. From this, we developed a specialized metadata vocabulary, to be used together with 
the Dublin Core metadata for characterizing the cases. Starting from that, either still-image based cases and digital slides may be easily described through RDF.

We claim that using RDF (with the proposed approach) in addition to any kind of case storage may give a novel form of interoperability for digital pathology cases, even in the lack of standards that currently characterizes telepathology.

\section{2}

\section{PIXEL-BASED SOFTWARE AUTOFOCUS FOR DIGITAL SLIDE ACQUISITION}

Della Mea V. (1), Viel F. (2), Beltrami C.A. (2)

(1) Inst. of Pathology, University of Udine, Italy, (2) Department of Mathematics and Computer Science, University of Udine, Italy

In the process of acquiring digital slides, a major requirement is the possibility of letting the apparatus (either robotized microscope or slide scanner) devoted to acquisition run in an unsupervised way for all the time needed for the acquisition of thousands of images. Among the critical issues, one is the autofocus, from which the image quality is strictly dependent.

Expensive research microscopes sometimes provide for an embedded autofocus system, which might be used for automatically focusing before acquisition of every image during the digital slide creation. However, such autofocus systems are usually designed for supporting the human user work, and may sometimes fail during a digital slide acquisition.

In order to overcome this, and also to exploit low-cost robotized microscopes, we developed an autofocus algorithm specifically aimed at bright-field microscopy, and unsupervised runs on whole glass slides.

Two aspects should be covered by an autofocusing algorithm: a focus measure and a search strategy. The former should be a measure of detail present in the images, while the latter is the method used in locating the position with higher focus measure.

In our approach, we used a dichotomous search algorithm based on the standard deviation of pixel brightness calculated on the green image channel (chosen among four tested measures). To this, a variable position space has been added in order to follow the variations in focus position throughout the slide, while avoiding the performance degradation given by a large position space.

The experiments made on histologic and cytologic samples allowed us to confirm the suitability of the proposed algorithm for the acquisition of digital slides.
73

THE IMPLEMENTATION OF AN IMAGE
CYTOMETER QUALITY ASSURANCE
SYSTEM USING STATISTICAL PROCESS
CONTROL ON IMAGE DATA OF THE
PRESS-PRO21 REFERENCE SLIDE

Chiu D. (1), Guillaud M. (2), MacAulay C. (3), Follen M. (4)

(1) British Columbia Cancer Research Centre, Canada, (2) University of Texas, USA, (3) MD Anderson Cancer Center, USA, (4) Perceptronix Medical Inc., USA

To enable reproducible and comparable clinical and research results from automated or semi-automated quantitative cytology and histology specimens, a rigorous quality assurance procedure is required. An Image Device Quality Assurance System was implemented at the British Columbia Cancer Research Centre and the University of Texas, MD Anderson Cancer Center for the purpose of monitoring data quality as part of an ongoing joint research effort. The QA system uses Statistical Process Control (SPC) methods applied to image data from periodic imaging of the PRESSPRO21 reference slide. This system monitors both semi-automated (histologic) and fully automated (cytologic) imaging systems. The System monitors field uniformity, short term and long-term system stability, and system linearity. The System consists of procedures and software. This presentation describes the main aspects of this quality assurance system applied across five systems over a twenty-month period. This work is funded by a grant from the National Cancer Institute and the National Institute of Health \#1PO1 CA82710-03.

\section{5}

\section{COMPUTING FRACTAL FEATURES FROM GRAY LEVEL IMAGES OF CELLS}

Albregtsen F. (1), Nielsen B. (1,2), Danielsen H.E. (2,3) (1) Department of Informatics, University of Oslo, Norway, (2) Division of Digital Pathology, Norwegian Radium Hospital, Norway, (3) Division of Genomic Medicine, University of Sheffield, England

Diagnostic estimates of the fractal dimension of gray level images of cells are often obtained by box counting or surface estimates at only a few resolutions. However, to obtain the fractal dimension, one must verify the existence of a linear relation in the log-log domain between e.g., surface area and resolution. This verification is often omitted. 
In our approach, we transform the 2D gray level images into $1 \mathrm{D}$ gray level signals. We then approximate the 1D gray level signal by polygonization with several tolerance values, T. For each tolerance value the total length, $\mathrm{S}(\mathrm{T})$, of the line segments that approximate the curve is found. For each cell nucleus we compute the slope of the best fitted straight line through three and three points in the $\log (\mathrm{T}), \log (\mathrm{S}(\mathrm{T}))$-domain. We store this as a Fractal Signature vector, FS(T).

For each tolerance value $\mathrm{T}$ we estimate the class conditional probability distributions of FS(T). We then compute the Class Difference Vector as well as the FS Class Distance Vector, between the classes. Using the Class Difference Vector as a binary decision function and the Class Distance Vector as a weight function, two adaptive FS features are obtained by a weighted summation of the FS vector values from different polygonization tolerances. Thus, we do not have to inspect for linearity in the $\log (\mathrm{T}), \log (\mathrm{S}(\mathrm{T}))$-domain.

\section{6}

\section{APPLICATION OF FRACTAL GEOMETRY TO THE STUDY OF BRAIN CORTEX}

Kalmanti E. (1), Haniotis V. (2), Maris T. (3)

(1) Department of Neurology Nikea General Hospital of Athens, Greece, (2) Department of Medical Physics University of Crete Medical School, Greece, (3) Department of Pathology University of Crete Medical School, Greece

In order to study the convolution pattern of cerebral cortex during aging and to compare it with brain atrophy we tried to standardize a method and applied fractal geometry. In a preliminary study a small number of patients of various ages. All subjects enrolled in this study underwent MR imaging on an 1.5 T MR system using standard quadrature RF head coil apparatus. A 3D multi-slice-single-echo GRE sequence endorsed with magnetization prepared prepulses (TR/TE/FA: $9.7 \mathrm{~ms} / 4 \mathrm{~ms} / 12^{\circ}$ ) was used. One single sagital 3D slab (160 mm thickness) with 64 partitions was obtained, thus giving $642 \mathrm{D}$ sagital slices of $2.5 \mathrm{~mm}$ effective thickness and no interslice gap. A rectangular FOV covering an area of $270 \times 236 \mathrm{~mm}^{2}$ was used. The image reconstruction matrix was $256 \times 224$ pixels respectively to the FOV dimensions, corresponding to a pixel matrix with square pixel dimensions $1.05 \times 1.05 \mathrm{~mm}$. One signal average and a small receiver bandwidth (195 Hz/pixel) were utilized. All 64 images (12 bit greyscale, DICOM-3 format) were transferred to a PC workstation where a 12 to 8 bit dynamic range com- pression using a best histogram equalization algorithm was performed for each image separately. Images were then stored utilising the standard 8 bit BMP format.

Left and right cerebral anatomy was recognized and manually extracted using standard contouring algorithms from a series of selected left and right cerebral parasagital slices. Automatic thresholding technique using a pixel value range (0-127) as 0 and a pixel value range (128-255) as 1 was applied in order to perform a series of a 2 bit black and white image for further fractal analysis. A standard box counting method was utilized using these images and a Fractal Dimension (FD) was calculated for each image. An average FD value obtained from the series of left and right cerebral parasagital slices was also calculated and utilized for inter-subject comparisons.

From our first results it appears that FD of cerebral cortex of normal individuals does not show significant differences.

\section{7}

\section{CLASSIFICATION OF HEREDITARY AND SPORADIC BREAST CANCERS BASED ON CLINICOPATHOLOGICAL DATA}

van der Groep P. (1), Bouter A. (1), Siccema T. (6), Krul M. (5), Menko F.H. (3), Michalides R. (4), van der Wall E. (2), van Diest P.J. (1)

Departments of (1) Pathology, (2) Medical Oncology, and (3) Clinical Genetics, VU University Medical Center, (4) Department of Tumor Biology, The Netherlands Cancer Institute, (5) NDDO Research Foundation, and (6) KiQ, Amsterdam, The Netherlands

Introduction: About 5\% of all the breast cancer cases are due to an inherited predisposition by germ line mutations in the BRCA1/2 genes. Screening for BRCA mutation in suspected carriers is however difficult and mutations may well be missed, which hampers genetic counselling. Additional features pointing to hereditary breast cancer are therefore useful.

Methods: Age of presentation, tumour size, mitotic index (MAI) and the expression of Ki67, p53, p21, p27, cyclin D1, cyclin A, estrogen (ER) progesterone (PR), Epidermal Growth Factor (EGFR), and HER-2/neu receptors by immunohistochemistry were compared between 27 proven BRCA1/2 mutation carriers and over 500 unselected controls. In addition, test cases at intermediate risk of hereditary disease based on familial history were evaluated.

Results: Hereditary breast cancers showed higher MAI, and Ki67/p53/cyclin A indices, more frequent 
EGFR overexpression, and lower ER/PR compared to the controls. Based on a discriminant function including age, Ki67 and EGFR, 96\% of the hereditary cases and $92 \%$ of the control cases were correctly classified. Most cases at intermediate risk of hereditary disease based on familial history could be classified with high probability as either hereditary or sporadic with this classification function.

Conclusion: Breast carcinomas can be classified as sporadic or as part of a hereditary syndrome with a high level of certainty using a discriminant function based on age, Ki67 and EGFR. This could be clinically useful to guide mutation analysis in families with borderline risk of hereditary disease.

\section{8}

\section{HIF-1 $\alpha$ /CAIX COEXPRESSION IN INVASIVE HUMAN BREAST CANCER}

van Diest P. (1), van Berkel M. (1), van der Groep P. (1), Shvarts A. (2), Bos R. (1), Greijer R. (2),

van der Wall E. (2), Harris A. (3)

Department of Pathology (1), and Medical Oncology (2), Free University Hospital, Amsterdam, The Netherlands, (3) Imperial Cancer Research Fund Molecular Oncology Laboratory and Angiogenesis Group, Institute of Molecular Medicine, John Radcliffe Hospital, Oxford, United Kingdom

Tumour hypoxia is one of the hallmarks of solid tumours, often associated with decreased efficacy of therapy. Furthermore, hypoxia is powerful trigger for angiogenesis and associated with tumour progression. In general cellular hypoxia triggers a broad response that is primarily mediated by the heterodimeric transcription factor hypoxia inducible factor (HIF-1). From our previous studies we concluded that the level of HIF- $1 \alpha$ increases during breast carcinogenesis, especially in poorly differentiated lesions, and is associated with poor prognosis in lymph node negative patients. Our previous studies further suggest that HIF-1 overexpression in breast cancer may also be oncogenerelated, as we have observed diffusely HIF- $1 \alpha$-overexpressing tumours without (hypoxic) necrosis.

Tumour cells may survive the hypoxic stress by creating an acidic environment that protects mitochondria. Carbonic anhydrases that reversibly convert carbon dioxide and water to carbonic acid, may play a role in this process. Interestingly carbonic anhydrase 9 protein (CAIX) was found to be expressed in various tumours and even more interestingly the expression of CAIX is regulated by HIF- $1 \alpha$, and has been shown to be associated with poor prognosis in breast cancer. Therefore, the aggressiveness of CAIX expressing tumours may be explained by HIF-1 $\alpha$ expression.

In the present study we examined the expression of CAIX and HIF- $1 \alpha$ in a group of 30 randomly selected invasive breast cancer cases by brightfield immunohistochemical single staining (of serial sections) and double staining (the CAIX and HIF1 $\square$ positive cases) using monoclonal antibodies to HIF- $1 \alpha$ and CAIX.

Having observed colocalization of HIF- $1 \alpha$ and CAIX in the single stained serial sections in many cases, we confirmed HIF-1 $\alpha$ /CAIX coexpression in the same cells by double staining, predominantly in perinecrotic areas (33\%). Rarely, expression of either HIF$1 \alpha$ or CAIX was seen in non-perinecrotic areas.

The results support the hypothesis that HIF-1 $\alpha$ expression is regulated by two different pathways (1) hypoxia mediated, visible as peri-necrotic HIF- $1 \alpha$ expression with concerted CAIX expression and (2) oncogene/ tumour suppressor gene regulated, that results in diffusely HIF- $1 \alpha$ expressing tumours. The absence of expression of HIF- $1 \alpha$ target genes in diffusely HIF- $1 \alpha$ expressing tumours suggests that these tumours may be less aggressive than those harboring necrosis. CAIX may be a more useful marker of tumor hypoxia than HIF-1 $\alpha$.

\section{9}

\section{COMBINATION OF \\ IMMUNOHISTOCHEMISTRY AND \\ MULTIPARAMETER FLOW CYTOMETRY \\ LEADS TO THE IDENTIFICATION OF A GROUP OF LOW PROLIFERATING HER2/NEU-OVEREXPRESSING BREAST TUMORS}

Leers M.P.G., Hoop J.G.M., Nap M.

Atrium Medical Center Heerlen, Department of Pathology, P.O. Box 4446, 6401 CX Heerlen, The Netherlands

Aim of the study: This study was designed to study the correlation between immunohistochemical (IHC) and multiparameter flow cytometric determination (MP-FCM) of Her2/neu, MP-FCM determined estrogen receptor-content (ER) and cell cycle characteristics (DNA ploidy and S-phase fraction (SPF)) in breast carcinomas.

Materials and Methods: For this purpose a series of routinely processed breast tumors of 200 patients were analysed using a recently developed technique for the 
isolation of single cells from paraffin embedded material. Single cells were immunostained simultaneously for cytokeratin and Her2/neu, or cytokeratin and ER. Finally, after DNA-staining samples were analysed on a DAKO Galaxy flow cytometer. Immunohistochemistry for Her2/neu was done on paraffin sections using the same antibody in an indirect streptavidinbiotin-peroxidase procedure. The immunohistochemical stained sections were scored on a scale of 0 (complete negative) to 3 (strong circumferential membranous staining).

Results: Her2/neu expression as measured by IHC showed $32 \%$ score $0,27 \%$ score $1,25 \%$ score 2 and $16 \%$ score 3 reactivity. The correlation between IHC and MP-FCM was significant $(r=0.87, p<0.0001)$. The majority of tumors (87\%) with IHC-scores 0 and 1 contained a MP-FCM determined Her2/neupositive epithelial cells of less than $25 \%$. It was noticed that Her2/neu-negative tumors with a diploid DNAcontent were almost all ER-positive (97\%). Her2/neunegative tumors with an aneuploid DNA-content were in $53 \%$ of the cases also ER-negative. On the other hand, a large fraction of the breast tumors with strong overexpression of Her2/neu ( $>50 \%$ Her2/neu-positive cells) were ER-negative (67\%). The proliferative activity (SPF) of the total epithelial compartment increased gradually when related to an increase in Her2/neuexpression and a simultaneous disappearance of ER. Regarding the SPF, two groups with different phenotypic aspects attracted our attention:

group $A \rightarrow$ tumors with a Her $2 /$ neu IHC-score 2 with a low ER-expression ( $<35 \% \mathrm{ER}+$ cells), high Her2/neu ( $>50 \%$ Her $2 /$ neu+ cells) and in 10 out of 11 cases aneuploid DNA-content, having an average SPF of $11 \%$ compared to $17 \%$ in other IHC-score 2 tumors.

group $B \rightarrow$ tumors with a Her $2 /$ neu IHC-score 3 with high ER-expression (mean ER+ cells of $62 \%$ ), moderate Her2/neu (mean Her2/neu+ cells of $49 \%$ ) and in 7 out of 9 cases diploid DNA-content, having an average SPF of $13 \%$, compared to $21 \%$ in remaining IHC-score 3 tumors.

Conclusions: The present study not only shows a strong correlation between IHC and MP-FCM, but in addition illustrates the discriminative power of MPFCM for the analysis of cell phenotypic markers in combination with DNA-bases cell cycle characteristics. A more precise identification of subgroups with co-expression of different receptors involved in therapeutic interventions, in combination with cell growth parameters may help in the selection of the most efficient application of individual therapeutic support.
80

\section{A COMPARISON OF THE} MULTIPARAMETER FLOW CYTOMETRIC STEROID HORMONE RECEPTOR ASSAY AND IMMUNOHISTOCHEMICAL RESULTS OF LARGE STUDIES IN THE LITERATURE

Leers M.P.G., Pannebakker M., Nap M.

Atrium Medical Center Heerlen, Department of Pathology \& Internal Medicine, P.O. Box 4446, 6401 CX Heerlen, The Netherlands

Introduction: Multiparameter DNA flow cytometric technique (MP-FCM) for steroid hormone receptor expression in breast carcinomas ${ }^{1}$ is an alternative for the quantification of hormone receptor-positive cells, which is routinely used in our institute for this purpose. In all cases a positive fraction of hormone receptor expressing epithelial cells was detected. This observation raised the question of defining a possible cut-off value for separation of receptor-negative and -positive tumors.

Materials and Methods: To establish a threshold of positivity, breast cancer data of our database ( $n=$ 345) were compared with a large immunohistochemical (IHC) study of Rhodes et al ( $n=7016$ breast carcinomas). ${ }^{2}$ In this study the frequency of receptor positivity was analysed by stratifying data according to patient age. It appeared that $35 \%$-receptor reactive epithelial cells by MP-FCM resulted in a similar distribution of ER- and PR-negative tumors according to age groups as compared with the Rhodes-study. This threshold was used to compare our MP-FCM data of tumor variants by hormone receptor expression with that of the National Cancer's Institute SEER-study (a study including 19541 patients). ${ }^{3}$ For this purpose, our data were restricted to women with node-negative breast cancer ( $n=169$ ). Standard tumor cell characteristics were analyzed by independent estrogen receptor (ER+ and ER-), independent progesterone receptor (PR+ and PR-), and joint ERPR expression (ER+PR+, ER+PR-, ER-PR+, and ER-PR-). The size of the fraction of ER (and/or) PR-expressing cytokeratin-positive cells determined by MP-FCM were compared with immunohistochemical data.

Results: When a threshold of $35 \%$ steroid hormone receptor positive epithelial (cytokeratin positive) cells was used, an acceptable match was found in the frequency of steroid hormone receptor positivity between the study of Rhodes et al and our MP-FCM data. Using the threshold of $35 \%$ receptor positivity, the relative sizes of tumor variants by expressing hormone recep- 


\begin{tabular}{|c|c|c|c|c|c|c|c|c|}
\hline & $\begin{array}{l}\text { Age } \\
\text { years }\end{array}$ & $n=$ & $\begin{array}{c}\mathrm{ER}+ \\
\%\end{array}$ & $\begin{array}{c}\mathrm{PR}+ \\
\%\end{array}$ & $\begin{array}{c}\mathrm{ER}+\mathrm{PR}+ \\
\%\end{array}$ & $\begin{array}{c}\text { ER-PR- } \\
\%\end{array}$ & $\begin{array}{c}\text { ER+PR- } \\
\%\end{array}$ & $\begin{array}{c}\text { ER-PR+ } \\
\%\end{array}$ \\
\hline \multirow[t]{3}{*}{ MP-FCM } & 35 to $<50$ & 31 & 18 & 19 & 19 & 19 & 14 & 23 \\
\hline & 50 to $<65$ & 56 & 31 & 30 & 30 & 40 & 36 & 35 \\
\hline & $\geqslant 65$ & 81 & 50 & 51 & 50 & 38 & 50 & 42 \\
\hline \multirow[t]{3}{*}{ SEER } & 35 to $<50$ & 2728 & 18 & 20 & 19 & 31 & 12 & 37 \\
\hline & 50 to $<65$ & 4718 & 31 & 31 & 31 & 33 & 31 & 34 \\
\hline & $\geqslant 65$ & 7612 & 50 & 48 & 49 & 33 & 56 & 27 \\
\hline
\end{tabular}

tors and age correlated well when comparing the multiparameter flow cytometric data with the SEER-study (see table).

Conclusion: MP-FCM is an alternative for IHC determination of ER and PR positivity. It is more objective and quantification can be done more appropriate. By comparing one's data with those from large data-collections in the literature, a threshold-\% can be defined for separation of steroid hormone receptornegative and -positive tumors.

\section{1}

\section{A STUDY OF APOPTOSIS IN APOCRINE METAPLASIA OF THE BREAST}

El-Ayat G. (1), Selim A.A. (1), Ghaly L. (2), Wells C.A. (1)

(1) Department of Histopathology, St Bartholomew's Hospital, London, EC1A 7BE, United Kingdom, (2) ICRF Laboratory of Skin Cancer, Royal London Hospital, London, E1, United Kingdom

Apocrine metaplasia (APM) of the breast is a common finding in breasts of post-menopausal women. During the past few years a number of reports have suggested a premalignant potential of some forms of APM. In a previous series of 63 cases, we reported that a subset of papillary APM was associated with cell cycle deregulation manifested by over-expression of cyclin D-1 and p21 which was associated with increased Ki-67 mean percentage positivity. We have used the TUNEL technique to determine the degree of apoptosis in 30 cases of APM including 25 cases showing papillary features and increased Ki-67 index. We have also examined the expression of the pro-apoptotic marker Bak and the anti-apoptotic markers Mcl-1 and Bcl-xl in these cases by immunohistochemistry. The TUNEL results show that APM shows an apoptotic index of less than $1 \%$ which is similar to that of normal breast epithelium. The increased mean $\mathrm{Ki}-67$ index of up to $5.5 \%$ seen with some of the cases showing papillary features is therefore not associated with increased apoptotic ac- tivity indicating a true proliferative capacity of these lesions. A constant cytoplasmic staining of APM for Mcl-1, Bcl-xl, and Bak indicates a maintained state of equilibrium between the pro-apoptotic and the antiapoptotic proteins in these cases. Our results suggest that APM is not an end stage of differentiation and that a subset of these metaplasias with papillary features are associated with increased proliferation that is not associated with an increased degree of cell death. These findings indicate a possible oncogenic capability.

83

EVALUATION OF THYMIDYLATE SYNTHASE PROTEIN EXPRESSION BY WESTERN

BLOTTING AND IMMUNOHISTOCHEMISTRY ON HUMAN COLON CARCINOMA XENOGRAFTS IN NUDE MICE

Montanaro L., Chillà A., Tosti E., Treré D., Ceccarelli C., Öfner D., Derenzini M.

Department of Experimental Pathology, University of Bologna, Italy

Thymidylate synthase (TS) is an enzyme necessary for the de novo synthesis of DNA in proliferating cells, as it catalyses the methylation of deoxyuridine monophosphate to thymidine monophosphate. TS is the target for fluoropyrimidine drugs like 5-fluorouracil which is the drug of choice for systemic therapy in colorectal cancer. In the present study we have investigated the relationship between TS protein expression, evaluated both by Western blot analysis and by immunohistochemistry, and growth rate in human colon xenograft tumors in nude mice. Human colon cancer cell lines were used to induce xenograft tumors and the tumor mass growth rate was calculated by measuring tumor size variations over time. TS 106 monoclonal antibody was used for both Western blot and immunohistochemical TS detection. Tumor cell growth fraction was measured by Ki67/MIB1 immunolabeling and tumor cell growth rate by evaluating the mean nucleolar size in silver-stained sections. TS expres- 
sion evaluated by Western analysis was related to both tumor mass growth rate $(p<0.001)$ and cell growth rate $(p=0.002)$, but not to cell growth fraction $(p=0.676)$. The degree of intensity of immunohistochemical staining showed only a trend to be associated with TS protein expression measured on Western blot, and was not related either to tumor mass growth or cell proliferation rate.

Our results demonstrated that TS expression evaluated by Western blot analysis is directly related to the tumor mass growth rate and questioned the use of the immunohistochemical approach to obtain precise quantitative information on TS expression in tumor samples.

\section{4}

\section{DETECTION OF PLASMA AND RENAL} VITAMIN E LEVELS IN LPS-INDUCED RAT RENAL INJURY

Kadkhodaee M., Gharibzadeh Sh., Ghasemi A., Shams S., Gol A.

Department of Physiology, Faculty of Medicine,Tehran Medical Sciences University, Tehran, Iran

Gram-negative bacterial lypopolysaccharide (LPS) release and subsequent septic shock is a major cause of death in intensive care unit. LPS is reported to increase nitric oxide (NO) production and oxygen-derived free radicals (OFR) formation in different organs. This study was designed to evaluate the role of iNOS (an inducible form of nitric oxide synthase) activity and OFR production in LPS-induced acute renal failure.

Measurement of vitamin $\mathrm{E}$ as the most important fatsoluble antioxidant, was used as a marker of tissue oxidative stress. LPS $(10 \mathrm{mg} / \mathrm{kg})$, L-iminoethyl Lysine (L-Nil, a specific inhibitor of iNOS activity, $3 \mathrm{mg} / \mathrm{kg}$, ip) and dimethylethiourea (DMTU, $500 \mathrm{mg} / \mathrm{kg}$, ip, a well-known OFR scavenger) were used.

Four groups of 8 rats were studied. One group received LPS, while a second group received LPS+ LNil. A third group received LPS+DMTU and a saline group, which served as a control. To evaluate renal function, plasma creatinine \& BUN were measured. HPLC and UV detection were used to measure plasma and tissue vitamin E levels.

LPS markedly decreased vitamin E contents of renal plasma and tissue $(p<0.05)$. Administration of L-Nil and DMTU prevented this reduction $(p<0.05)$. Inhibition of iNOS activity by L-Nil attenuated renal dysfunction, suggesting involvement of an overproduction of NO by iNOS in LPS-induced renal failure.
85

VEGF, CD34 AND MICROVESSEL DENSITY IN THE GANGLIONEUROBLASTOMA GANGLIONEUROMA SEQUENCE

Kreczy A., Abrederis K., Gschwendtner A., Meister B. Department of Pathology, University of Innsbruck, Austria

We investigated the significance of the angioneogenesis in the neuroblastoma-/ganglioneuroma-sequence using 50 tumorblocks of 22 consecutive patients of the last 10 years from the Department of Pathology of the University of Innsbruck. Additionally, we investigated the tumor induced neoangiogenesis in 28 specimens of the bone marrow of 15 patients. After applying immunohistochemical stainings for VEGF and CD34, the slides were evaluated using quantitative morphometrical methods. Due to the small numbers of cases in the different groups, only descriptive statistical methods were applicable. In neuroblastomas, VEGF expression and microvessel density correlated with tumor stage, metastasis, ploidy and N-myc amplification, whereas the other prognostic factors as Ipdeletion, HVA, VMA, Ferritin and NSE did not correlate. Bonemarrow affected by the metastasis of neuroblastoma or other diseases showed a threefold microvessel density when compared with normocellular marrow. Ganglioneuroblastomas and ganglioneuromas showed higher vessel densities than neuroblastoma indicating, that VEGF and microvessel density are not independent prognostic factors. The fact, that at the same time ganglion cells in ganglioneuroma express higher levels of VEGF than neuroblastoma cells indicates the importance of antiangiogenetic factors deriving from schwann-cells and mature stromal cells.

\section{6}

ULTRASTRUCTURAL MORPHOMETRY AND PROGNOSIS OF SOLID TUMORS: A REVIEW

Lloreta-Trull J., Conde G., Ferrer L., Bielsa O.,

Díaz E., Iglesias M., Corominas J.M., Serrano S.

Department of Pathology, Hospital del Mar-IMASIMIM, Universitat Pompeu Fabra, Barcelona, Spain

Quantification of morphologic features has been shown to be a reproducible method for objective prognostic stratification of solid tumors, particularly carcinomas. Many ultrastructural features of tumor cells are related to their behavior. Quantifying such features could help in defining specific prognostic categories. 
We have morphometrically investigated ultrastructural nucleolar features (size, contact with nuclear membrane, number of nucleoli per nucleus) in renal cell carcinoma ( $n=100)$, node-negative infiltrating duct carcinoma of breast ( $n=43$ ) and classical medullary carcinoma ( $n=10$ ), as well as cytoplasmic or cell surface features in both breast cancer groups and in nasal neuroendocrine carcinomas $(n=24)$ (number of desmosomes, number of dendritic or axonal cell processes, respectively).

Nucleolar size has been fairly precise in the stratification of renal cell carcinoma into low and high grade categories. This parameter is also predictive of stage and histological grade. In infiltrating duct carcinomas, the presence of more than 3 nucleoli per nuclear section and their contact with the nuclear membrane are excellent prognostic indicators of recurrence, with highest predictive positive and negative values and highest sensitivity than tumor size. Number of desmosomes per cell membrane unit is a good indicator for metastatic medullary carcinoma but not for infiltrating duct carcinoma. Finally, the presence of a minimum number of cell processes in a nasal neuroendocrine tumor is associated with excellent prognosis, even when the neuroblastic differentiation can only be appreciated with the electron microscope.

In conclusion, measurement of certain biologically meaningful ultrastructural features of tumors proves to be very helpful in specific situations. Electron microscopy facilities are not available to all institutions, but centralized laboratories may provide service to many hospitals. In addition, ultrastructural morphometric assessment helps in the identification of relevant features that can be included in light microscopic grading systems with the use of refined image analysis and improved light microscopic techniques.

\section{7}

\section{A QUANTITATIVE APPROACH OF FIBROSIS IN IDIOPATHIC PULMONARY FIBROSIS/USUAL INTERSTITIAL PNEUMONIA}

Koutsopoulos A.V., Stathopoulos E.N., Kafusi M., Chaniotis V., Buros D., Delides G.

Department of Pathology, University of Crete, Medical School, Greece

Idiopathic pulmonary fibrosis is a distinct clinicopathologic entity with poor prognosis and no effective therapy. The histological features are well established and consist of architectural destruction with patchy distribution of fibrosis, often with honeycombing, mild to moderate interstitial inflammation, type II cell hyperplasia, intraalveolar macrophage accumulation, marked smooth muscle metaplasia/hyperplasia and frequent subpleural or paraseptal accentuation. The fibrotic zones show temporal heterogeneity, with areas of dense collagen deposition and scattered fibroblastic foci.

The pulmonary destruction leads to insufficient lung function and impaired gas exchange, due to decreased alveolar surface and extensive thickening of alveolar septae.

In order to estimate the extension and severity of fibrosis, we performed quantitative morphometric analysis in 12 lung biopsy specimens (VATS), from patients with I.P.F./U.I.P. and 12 specimens of "normal lung". The sections were stained histochemically for collagen, and quantitative analysis was performed using image analysis software developed for this propose by FORTH. The degree of fibrosis was expressed as the percentage of fibrosis to total cut surface of each specimen included in this study. The possible correlation between fibrosis and forced vital capacity (FVC) was investigated.

Our findings indicate a correlation between the degree of fibrosis and the FVC.

\section{8}

CELLULAR and MOLECULAR

CHARACTERIZATION OF CIRCULATING BREAST TUMOR CELLS AFTER LEUKAPHERESIS IN BREAST CARCINOMA (BC) PATIENTS BY RT-PCR, IMMUNOHISTOCHEMISTRY (IHC), LASER SCANNING CONFOCAL MICROSCOPY (LSCM), LASER SCANNING CYTOMETER (LSC), and FLUORESCENT IN SITU HYBRIDIZATION (FISH)

Bhattacharya S. (1), Juan G. (1), Hudis C. (2), Scher H.I. (2), Rosen N. (2), Hutchinson B. (1), Salazar P.A. (1), Desai S. (1), Ladanyi M. (1), Cardon-Cardo C. (1), Ghossein R.A. (1)

Department of (1) Pathology, (2) Medicine, Memorial Sloan-Kettering Cancer Center, New York, NY, USA

Circulating BC cells were isolated by immunomagnetic isolation procedure from three Leukapheresis samples from two highly progressive metastatic BC patients (one patient with pre- and post chemotherapy products). The mRNA isolated from the enriched frac- 
tion was tested for the presence of Mammaglobin and Cytokeratin (CK-19) by Nested RT-PCR. Magnetic beads were removed from the rest of the epithelial enriched fraction; the cells cytospun, fixed and evaluated either for Immunohistochemistry (IHC) for Cytokeratin \& Her2-neu; or for LSC and LSCM, for the simultaneous detection of Her2-neu, Epidermal Growth Factor (EGFR) \& Ki-67, Propidium Iodide (PI) and DAPI. Samples were also processed for the detection of Her2neu amplification by FISH. Metastatic BC to liver from one patient and $\mathrm{BC}$ cell line, $\mathrm{MCF}-7$, served as positive controls.

Results: All the samples were negative for CK-19; but positive for Mammaglobin mRNA, as detected by Nested RT-PCR. The metastatic breast tissue had a high expression of Her-2 neu (3+, by IHC). The MCF7 and the metastatic tissue showed strong membranous EGFR \& Her2-neu, and nuclear Ki-67 positivity by LSCM. The patient samples were negative by LSC for Her2-neu and EGFR. LSCM showed nuclear Ki67, cytoplasmic Her2-neu, \& membranous EGFR fluorescence in cells that were morphologically consistent with leukocytes. In one patient sample, FISH showed Her2-neu amplification (ratio: 1.7).

Conclusion: This study demonstrates the importance of a comprehensive technical approach to the study of Circulating Tumor Cells that includes morphological analysis and a multiple marker molecular assay.

\section{9}

\section{MALIGNANCY ASSOCIATED CHANGES AS A POSSIBLE SURROGATE ENDPOINT BIOMARKER IN PROSTATE CANCER}

Gschwendtner A., Mairinger T., Palcic B., Mikuz G., Bartsch G.

Department of Pathology, University of Innsbruck, Austria

Introduction: Prostate cancer is the most frequently diagnosed cancer among men in the western industrialised world. Modern PSA screening has led to a dramatic increase in the detection of early stage disease, however biological behaviour of these tumours is highly variable. If left untreated only a small proportion of patients are at risk for tumour progression and death. Therefore new surrogate endpoint biomarkers are urgently needed that would allow us to stratify patients for treatment. We previously demonstrated that prostate cancer induces submicroscopic textural changes in ostensibly normal cells growing in the vicinity of the malignant cells. We studied these cyto- logic alterations also known as malignancy associated changes (MAC) as a biomarker in prostate cancer.

Material and Methods: MAC was assessed as a biomarker in 79 patients following prostatectomy. To determine MAC, high resolution image cytometry was performed on at least 1000 normal appearing cells near from the malignant cells for each prostatectomy specimen. Using the previous established classifier a MAC score for every single case could be established. Tumor stage, Gleason grade, and DNA ploidy were also determined from the prostatectomy specimens. The MAC score was than correlated with these parameters.

Results: MAC was detected in $40-100 \%$ of normal appearing cells adjacent to the malignant cells. The MAC score was significantly correlated with tumour stage $(p \leqslant 0.001)$, Gleason score $(p \leqslant 0.01)$, and DNA ploidy ( $p \leqslant 0.01$ ). In all cases, nondiploid tumors were associated with a higher MAC score than diploid tumors allowing separation of the two groups with a sensitivity of $88 \%$ and a specificity of $83 \%$, using an arbitrary threshold.

Conclusions: High resolution image cytometry can reliably and reproducibly identify MAC in normal appearing cells near prostate cancer. MAC scores were significantly correlated with Gleason grade, tumor stage, and DNA ploidy. Our results suggest MAC may represent a valuable biomarker that can measure biologic potential of prostate cancer before treatment.

\section{1}

\section{SMALL PROSTATE CANCERS ARE TETRAPLOID}

Gschwendtner A., Mairinger T., Rogatsch H., Horninger W., Bartsch G.

Department of Pathology University of Innsbruck, Austria

Introduction: The aim of the following study was to evaluate, if small prostate cancers with low PSA levels and small tumor volume exhibit the same features as prostate cancers with higher tumor volumes in terms of ploidy and proliferation index.

Materials and Methods: 22 radical prostatectomy specimens with a tumor volume of less than 1cc were evaluated in terms of tumor volume, ploidy and proliferation index. All these patients were part of the Tyrol PSA Screening Program and underwent radical retropubic prostatectomy at the University Hospital of Innsbruck.

All 22 prostatectomy specimens were inked and fixed in $10 \%$ neutral buffered formalin for 48-96 hours. Sub- 
sequently the prostates were serially sectioned. Cancer volume in the prostatectomy specimens was determined from an ink tracing on each slide of the exact tumor outline as seen on microscopic inspection at low magnification $(40 \times)$. With a personal computer with digitizing pad, the area of tumor at each level at the section was determined.

Tumor ploidy was determined by means of DNA imaging cytometry using single cell preparations. In addition proliferative activity was assessed by means of KI67 (MIP-1) labeling index.

Results: The mean age of this study cohort was 59.6 years (range: 43-68), all 22 prostatectomy specimens evaluated had a tumor volume of less than $1.0 \mathrm{cc}$.

$36 \%$ of the specimens showed tetraploid DNA distribution, with a mean KI-67 index of 7.80. In the diploid cancer group the mean KI-67 index was 4.08. The difference between those 2 indexes was statistically significant.

Ploidy correlated well with $\%$ free PSA and the KI 67 index but there was no correlation with total PSA, Gleason Score and tumor volume.

Moreover, more than $50 \%$ of tetraploid tumors were found in lesions with tumor volumes of less than $0.5 \mathrm{cc}$. Discussion and Conclusions: DNA ploidy and cell proliferation factors such as $\mathrm{Ki} 67$ are predictive factors in prostate cancers and are associated with more aggressive prostate cancers and worse patients outcome. It was shown that aneuploidy may be used as a prognostic marker in locally advanced stage $\mathrm{C}$ prostate cancer and that it occurs in a high frequency in prostate cancers with advanced stages and strongly correlate with high Gleason Score.

In our study we could show that $36 \%$ of prostate cancers with a tumor volume of less than $1.0 \mathrm{cc}$ exhibit tetraploid DNA distribution. All these cancers also showed an increased KI 67 index. On the basis of these findings we conclude that in spite of small tumor volume prostate cancers can exhibit an aneuploid DNA status and high proliferative activity suggesting a more aggressive potential that does not justify to regard them as clinically insignificant cancers.

\section{5}

MYOCARDIAL FLUORESCENCE

MEASUREMENTS AS

MORPHOPATHOLOGICAL CRITERIA FOR DETERMINING THE DURATION OF MYOCARDIAL INFARCTION

Tsiple I., Anestiadis V.

Centre of Pathobiology and Pathology, Academy of Sciences, Chisinau, Moldova
The study aimed towards finding new criteria for the determination of the duration in time of myocardial infarction (MI). 52 hearts from persons deceased from MI, in which MI beginning was documented clinically and by ECG, were selected. 30 hearts of persons deceased almost instantaneously from traumas served as controls. In each case we did 1000 measurements of myocardial primary fluorescence (MPF) at the wavelength of $360 \mathrm{~nm}$, both in the necrotic (ischaemic) zone (NZ) and in extrainfarctic zones (EZ). During 8-12 hrs from MI beginning, the MPF decreased concordantly in both zones, being $89.6 \pm 2.3 \%$ of controls. At 24 hrs after MI onset MPF in NZ was $78.8 \pm 4.3 \%$, in EZ $484.4 \pm 3.1 \%$ of controls. Minimal NZ MPF was at $4-5$ th and $9-10$ th days $(66.3 \pm 2.5 \%$ and $62.6 \pm 2.1 \%$ of controls, $p<0.001)$. EZ MPF at 4-5th days was $77.4 \pm 3.5 \%$, at $9-10$ th days $82.3 \pm 2.2 \%$ and at 14-15th days $85.7 \pm 2.8 \%$, remaining even up to 3035 th days of MI $7.3 \pm 0.8 \%$ below controls. MPF variations in time were approximated by splines. By solving a system of equations, which included formulae for EZ and NZ MPFs, it was possible to determine the time elapsed from the MI onset, with an accuracy of $9.6 \mathrm{hrs}$.

\section{Posters}

P1

INCREASED MORTALITY RATE DUE TO ACCELERATED TELOMERE SHORTENING IS RESPONSIBLE FOR PROLIFERATIVE CHANGES IN DYSKERATOSIS CONGENITA CELL LINES

Montanaro L., Chillà A., Trerè D., Tazzari P.L., Derenzini M.

Department of Experimental Pathology, University of Bologna, Italy

$\mathrm{X}$-linked dyskeratosis congenita is a rare inherited disorder mainly characterized by progressive changes in proliferating epidermal, mucosal and bone marrow tissues that commonly emerge after ten years of life. It is caused by mutations of the $D K C 1$ gene which codes for dyskerin, a protein that may play a role in ribosomal biogenesis and in telomerase complex stabilization. In order to verify whether the defects of proliferating tissues observed in dyskeratosis congenita are due to an altered ribosome synthesis, we studied ribosomal biogenesis in relation to cell proliferation in two lymphoblastoid cell lines from dyskeratosis congenita 
patients and in one control line. We observed that in the dyskeratosis congenita cell lines the rRNA transcription and maturation and proliferative capability remained unimpaired. However, increasing the number of cell cycles lead to a steep rise in the apoptotic fraction of dyskeratosis congenita cells which is not observed in controls. Moreover, the increase in the cell divisions lead also to a very rapid decrease in the telomere length in dyskeratosis congenita cells, while control cells were characterized by a constant telomere length. These findings demonstrate that while dyskeratosis congenita cell lines do not display proliferation defects, they do show progressively increasing levels of apoptosis in relation to the number of cell divisions associated to a premature telomere shortening. This concept is consistent with 1) the delayed onset of dyskeratosis congenita proliferating-tissue defects which do not emerge during embrional development, as would be expected with ribosomal biogenesis alterations and 2) with the increasing severity of the proliferating-tissue defects over the time.

\section{P2}

\section{NUCLEOLAR HYPERTROPHY CORRELATES WITH HEPATOCELLULAR CARCINOMA DEVELOPMENT IN LIVER CIRRHOSIS DUE TO HBV INFECTION}

Trerè D., Borzio M., Morabito A., Roncalli M., Derenzini M.

Department of Experimental Pathology, University of Bologna, Italy

The aim of the present study was to define the value of the percentage of hepatocytes showing nucleolar hypertrophy as indicator of hepatocellular carcinoma (HCC) development in liver cirrhosis with different etiology. One hundred and eleven cirrhotic patients were studied with a mean follow-up of 83.3 months (range 8-201 months). For each case, nucleoli were visualized in histological sections of the liver biopsies by selective silver-staining, and their size objectively evaluated by image cytometry. Nucleolar hypertrophy was defined using a cut-off value of $7 \mu \mathrm{m}^{2}$ and the nucleolarindex was obtained calculating the number of hepatocytes disclosing a nucleolar area $\geqslant 7 \mu \mathrm{m}^{2}$. During the observation time, HCC was diagnosed in 39 patients with an annual incidence of $5.06 \%$. The incidence rate of $\mathrm{HCC}$ was significantly greater in patients with nucleolar-indexes $\geqslant 2.5(n=83)$ than in patients with nucleolar-indexes $<2.5(n=28)(16.49 \% /$ year vs. $3.41 \% / y e a r$, respectively; $p<0.0001)$. The capac- ity of the nucleolar-index to predict HCC development was separately tested in groups of patients divided by viral infection. Within HBsAg-positive patients, great nucleolar-index values were found to significantly discriminate those cases at higher risk of HCC onset ( $p=0.0006)$. Among patients with HCV infection, nucleolar-index values $\geqslant 2.5$ were associated with a greater risk of HCC development, but the difference in the cumulative probability between groups with nucleolar index $\geqslant 2.5$ and $<2.5$ was not statistically significant $(p=0.0944)$. Our results have demonstrated that, in HBV related cirrhosis, the percentage of hepatocytes with nucleolar hypertrophy represents a powerful tool for defining higher risk of HCC development. On the other hand, the capacity of this parameter to predict $\mathrm{HCC}$ onset in HCV related cirrhosis seems to be lower.

\section{P3}

\section{INTEGRATED TELEMEDICINE NETWORK IN} ONCOLOGY

Vári S.M.D. (1), Debreczeni K. (2), Cserneky M. (2), Godó F. (3), Brugal G.M.D. (4)

(1) VARIMED Ltd., (2) Semmelweis University Budapest, Hungary, (3) Q.Med., (4) Joseph Fourier University, Grenoble, France

Introduction: The European Commission sponsored BEPRO (IST-2000-25252) project aims to provide reference cancer centres with resources to extend the pilot use of results from previous European projects in Information Technologies for Health. Partners deploy IT applications, provide appropriate training to the end users and integrate those IT into the daily practice for the physicians.

Results: The BEPRO Project demonstrates best practice in action enabled by the use of innovative information the computer technology (ICT) applications in Oncology. The developed co-operative services allow sharing experience and reaching consensus at interapplication and transnational levels. Participants are dealing with data exchange procedures based on XML and DTD kept at the most generic pertinence levels dealing with state-of-the-art data exchange technologies independently from medical applications.

Conclusions: The BEPRO carries out safe integration of medical best practices in several respects: reference diagnostics, consensus on electronic forms and remote data entry to epidemiological centres and clinical trials coordinators, guidelines and continuous medical education. The use of ICT impact the best medical practice 
is still a challenge and a prerequisite for cost-benefit analysis regarding social and medical interests.

\section{P4}

\section{QUANTIFICATION OF MICROVESSELS USING COLOR IMAGE ANALYSIS}

Choi H.J. (1), Choi I.H. (1), Hwang H.G. (1), Cho N.H. (2), Choi H.K. (1)

(1) Medical Image Technology Laboratory, School of Information and Computer Engineering, Inje University, Korea, (2) Department of Pathology, Yonsei University, Korea

Angiogenesis is the formation of new capillaries from the existing vascular network and is essential to tumor growth and metastasis. In angiogenesis, Microvessel density (MVD) is used as the prognostic factor, because it has the significant correlation to progression of tumor. The purpose of our study is to quantify microvessel density using computerized color image analysis.

In our study, microvessels were segmented by the color image segmentation method based on color feature space clustering. First, 100 pixels were selected from microvessel and background regions in an image respectively. 6 color features (R, G, B, H, S, I) were extracted from the selected pixels. In the next place, 3 classifiers were created using multivariate discriminant analysis (MANOVA): the first classifier was made of R, G, B feature vector, the second classifier was made of $\mathrm{H}, \mathrm{S}, \mathrm{I}$ feature vector and the third classifier was made of their combination feature vector. Finally, each classifier was verified on a real test image. And the area proportions of the segmented microvessels were calculated and compared.

As the result of our study, we obtained microvessel density area $7.9121 \%, 9.1770 \%$ and $7.9742 \%$ for each of 3 classifiers. We found that the obtained result by RGB feature vector classifier has a good correlation with pathologists' opinion.

\section{P5}

\section{GRANULOSA-STROMAL AND}

SERTOLI-STROMAL CELL TUMORS OF THE OVARY: IMMUNOHISTOCHEMICAL STAINS FOR MELAN A AND CALRETININ AND FLUORESCENCE IN SITU HYBRIDIZATION (F.I.S.H.) FOR CHROMOSOMES 12 AND 7

Kostopoulou E., Agelidou S., Aslanidou E., Leontsini M.

Pathology Department, Hippokrateion Hospital of Thessaloniki, Greece
Background: Sex cord-stromal tumors account for 8 per cent of primary ovarian neoplasms and often pose diagnostic problems, due to their wide spectrum of histologic patterns. In recent years immunohistochemical stains can be of help in these problematic cases. The expression of calretinin, a calcium-binding protein, and staining for A103, an antibody for melan A, have been evaluated in several types of tumors. Additionally, trisomy 12 has been reported as a common finding in granulosa-stromal cell tumors; other trisomies have been reported rarely. The aim of the present study was to examine granulosa-stromal and Sertoli-stromal ovarian tumors with antibodies for calretinin and melan $\mathrm{A}$ and for the presence of trisomy 12 and 7 by F.I.S.H.

Methods: 7 granulosa cell tumors, 7 Sertoli-Leydig cell tumors, and 4 sclerosing stromal tumors were immunostained for calretinin (5A5, Novocastra, 16h, $4^{\circ} \mathrm{C}$ ) and A103 (Novokastra). Fifteen cases of carcinomas (ovarian and endometrial) and 7 endometrial or endometrioid stromal sarcomas were immunostained for comparison. $\alpha$-satellite probes (Appligene-Oncor) were used for F.I.S.H.

Results: 7/7 and 5/7 Sertoli-Leydig cell tumors were positive for calretinin and A103, respectively. Expression of calretinin was also observed in 5/7 granulosa cell tumors and 3/4 sclerosing stromal tumors. A103 immunostain was positive in 4/7 granulosa cell tumors and 4/4 sclerosing stromal tumors. Only one case of ESS was positive for calretinin. Trisomy 12 was found in $3 / 5$ granulosa cell tumors and $3 / 3$ sclerosing stromal tumors.

Conclusions: Our findings confirm the utility of calretinin and A103 as part of a panel of antibodies in differentiating ovarian tumors of the above group from their mimics. The percentage of positivity differs among the few reported series. Trisomy 12 is a common finding in granulosa cell tumors and sclerosing stromal tumors.

\section{P6}

\section{CLASSIFICATION OF CELL AND TISSUE IMAGES IN BREAST TUMOR}

Hwang H.G. (1), Choi H.J. (1), Choi I.K. (1), Yoon H.K. (2), Nam S.H. (3), Choi H.K. (1)

(1) School of Information and Computer Engineering, Inje University, Korea, (2) Department of Pathology, Inje University, Korea, (3) Department of Biomedical Engineering, Inje University, Korea

In this paper, we described three classification algorithms to classify breast lesion of ductal organ Be- 
nign(benign ductal hyperplasia), DCIS(ductal carcinoma in situ), CA(carcinoma, invasive ductal carcinoma). The general approach for a creating classifier is composed of 2 steps: feature extraction and classification. Above all, feature extraction for a good classifier is very significant, because the classification performance depends on the extracted features. Therefore, in the feature extraction step, we extracted morphology features including the shape, and size of nuclei and texture features. The internal structures of the tumor are reflected from wavelet transformed images with $10 \times$ and $40 \times$ magnification. Particularly, to find the correlation between correct classification rates and wavelet depths, we applied 1, 2, 3, 4, 5 and 6-level Haar wavelet transforms and Dauchies wavelet transformation the images and extracted texture features from the transformed images. The used morphology features are area, perimeter, width of $X$ axis, width of $Y$ axis and circularity. The texture used features are entropy, energy, contrast and homogeneity. In the classification step, we created three classifiers from each of extracted features using discriminant analysis. The first classifier was made by morphology features. The second and the third classifiers were made by texture features of wavelet transformed images with $10 \times$ and $40 \times$ magnification. Finally, we analyzed and compared the correct classification rate of the three classifiers. In this study, we found that the best classifier was made by texture features of 2-level Haar wavelet transformed images with $10 \times$.

P7

\section{DNA IMAGE ANALYSIS CYTOMETRY AND Bcl-2 PROTEIN EXPRESSION IN PRIMARY NON-SMALL CELL LUNG CARCINOMA}

Maounis N. (1), Chorti M. (1), Palamidas F. (1), Ellina E. (1), Blana A. (1), Legaki S. (1),

Tsakalou G. (1), Markidou S. (2)

(1) Sismanoglion General Hospital, (2) Saint Savas Hospital, Athens, Greece

The aim of this study was to assess the association between DNA ploidy and bcl-2 protein expression in Non-Small Cell Lung Carcinoma (NSCLC).

In 52 NSCLC the DNA measurement was performed by image analysis cytometry on Feulgen stained imprints taken immediately after the surgical removal of the tumor using the SAMBA image analysis system. Furthermore, the expression of the bcl-2 protein, as apoptotic marker, was studied performing immunohis- tochemistry on paraffin sections. Our results was related to various clinical or pathological parameters (patient's age, sex, the stage of the disease and the tumor type, size and differentiation).

DNA measurement showed a prevalence of aneuploidy $(65 \%)$ and $53.8 \%$ of our cases had $5 \mathrm{cER}>5 \%$. Our results revealed statistically significant correlation of ploidy with stage ( $p=0.047)$, aneuploidy being more frequently observed in high stage. In addition 5cER showed statistically significant dependence with tumor differentiation. Furthermore $5 \mathrm{cER}$ was associated with bcl-2 $(p=0.05) .5 \mathrm{cER}<5 \%$ was more frequently observed in positive bcl-2 cases (67.9\%) whereas $5 \mathrm{cER}>\%$ is more frequently observed in negative bcl-2 (62.5\%) cases.

In conclusion our findings suggest that image analysis cytometry and expression of bcl-2 can be a useful tool in the understanding of the biological behaviour of patients with NSCLC.

\section{P8}

\section{PROGNOSTIC SIGNIFICANCE OF DNA PLOIDY IN SURGICALLY TREATED NON-SMALL CELL LUNG CARCINOMAS}

Maounis N. (1), Palamidas F. (1), Delaportas G. (2), Legaki S. (1), Ellina E. (1), Blana A. (1), Chorti M. (1), Vaida S. (2), Markidou S. (2)

(1) Sismanoglion General Hospital, (2) Saint Savas Hospital, Athens, Greece

The aim of this study was to evaluate the role of DNA ploidy as a prognostic factor in Non- Small Cell Lung Carcinoma (NSCLC) and its relation to various clinicopathological parameters.

In a prospective study, 96 patients who underwent surgery because of NSCLC were investigated as follows: tumours were classified as squamous cell carcinoma (SCC) or adenocarcinoma, tumour differentiation, nodal status, size and pathological TNM staging (WHO) were evaluated. Additionally, all cases were classified in respect to their pathological TNM staging (WHO). DNA image analysis cytometry was performed on imprints, taken immediately after surgery, to determine DNA ploidy using the SAMBA system. DNA index and $5 c$ exceeding rate $(5 \mathrm{cER})$ were analyzed and the histograms were classified as near diploid and aneuploid. The median follow - up time of surviving patients was 26 months.

Out of the 96 cases 49 were SCC and 47 adenoCa, 54 cases $(56.3 \%)$ were stage I, $16(16.6 \%)$, stage II and 26 (27.1\%) stage IIIA. 
Ploidy was significantly associated with tumour differentiation, stage and size. More specifically, aneuploidy as compared to diploidy was more frequently observed in high stage $(p=0.002)$, in tumour size $>4 \mathrm{~cm}$ $(p=0.015)$ and in poor differentiation $(p=0.005)$. $5 \mathrm{cER}>5 \%(p=0.010)$ was associated with tumour size $>4 \mathrm{~cm}(p=0.010)$ and poor differentiation $(p=0.001)$. Survival analysis revealed that aneuploid cases had poor survival estimates compared to diploid cases $(p=0.0001)$. 5 cER $>5 \%$ was also associated with poor prognosis $(p=0.001)$.

In conclusion, DNA ploidy may identify individuals whose resectable NSCLCs have a more aggressive tumour behavior and thus may help to set new guidelines for adjuvant therapy.

\section{P9}

\section{THE EFFECT OF PREOPERATIVE CHEMOTHERAPY ON PLOIDY AND KARYOMETRIC PARAMETERS IN BREAST CARCINOMA}

Markidou S. (1), Maounis N. (2), Karatzia D. (1),

Georgoulakis J. (1), Misitzis J. (1),

Papaevangelou M. (1), Delaportas G. (1), Garoufalli A. (1), Karydas I. (1)

(1) Saint Savas Hospital, (2) Sismanoglion Hospital, Athens, Greece

The effect of preoperative chemotherapy on ploidy and morphometric parameters in breast carcinoma was the object of this study.

A group of 50 patients with operable breast cancer was studied. DNA ploidy and nuclear area were measured by image cytometry before and after chemotherapy using the SAMBA system, on Feulgen smears of breast FNA and imprints of the resected tumor for each patient. The DNA histogram, karyometric parameters (mean nuclear area (MNA) and nuclear area SD) of the FNA material were compared to those obtained from the imprint of the resected tumor after 3 cycles methotrexate/mitoxantrone chemotherapy.

Thirty tumors were aneuploid, 3 tetraploid and 17 diploid. The tumors of 31 patients did not respond to chemotherapy (no change in tumor size), 17 showed partial response and 2 cases total regression. Comparison of pre- and post chemotherapy DNA histograms showed in most of the cases no change in ploidy status. In two cases the diploid peak disappeared after chemotherapy. In the first case a diploid histogram changed into tetraploid. In the second case of an aneuploid histogram with two peaks, a diploid and an ane- uploid, the diploid peak disappeared after chemotherapy. In a third case one of the two aneuploid peaks disappeared. There was no correlation between the type of the histogram and response to chemotherapy. MNA and nuclear area SD did not show significant differences correlated with ploidy status or treatment response.

In conclusion preoperative chemotherapy did not have any significant effect on ploidy status or nuclear parameters. Therefore the type of DNA histogram obtained preoperatively on FNA material does not seem to provide any predictive information concerning the chemotherapy treatment response.

\section{P10}

THE EXPRESSION OF CD95 APOPTOTIC

MARKER IN PERICARDIAL FLUID OF PATIENTS OPERATED FOR CORONARY ARTERY BYPASS GRAFTING.CORRELATION WITH CLINICAL PARAMETERS

Apostolakis E. (1), Ellina E. (2), Maounis N. (2), Chorti M. (2), Dedeelias P. (1), Koletsis E. (1), Blana A. (2)

(1) Cardiovascular Surgery Department of "EVAGELISMOS" Hospital, (2) Cytology Department of Sismanoglion Hospital, Athens, Greece

Apoptosis is an active - gene regulated form of cell death different from necrosis.

Data suggests that apoptosis may play a role in the pathophysiology of coronary artery disease (CAD). It is also known that factors produced by the heart are accumulated at high concentrations in pericardial fluid inducing apoptosis.

The aim of the study was to assess the expression of CD95 in cytology samples obtained from pericardial fluid of 31 patients ( 20 men and 11 women) with coronary artery disease who underwent operation for coronary artery bypass grafting. The pericardial fluid was collected at the beginning of the operation, following the opening of the pericardium. Samples with mesothelial cells were investigated immmunohistochemically using monoclonal antibody CD95. The expression of CD95 was related to the following clinical parameters: sex, age $(>65)$, diabetes mellitus, chronic obstructive lung disease, history of myocardial infarction, clinical stage of coronary artery disease, number of diseased coronary arteries, proximal stenosis of right coronary artery (RCA) or left anterior descending artery (LAD), ejection fraction of left ventricle, unstable angina, and coexistence of any heart valve disease. 
Twenty three out of 31 examined samples were positive $(74.19 \%)$. Statistical analysis using the chi-square test revealed statistically significant correlation of the CD95 expression and both the 3 vessels CAD $(p<$ $0.005)$ and the proximal stenosis of LAD $(p<0.001)$. In conclusions, our results indicate that the intensive expression of CD 95 in pericardial fluid may indicate a relationship between apoptosis and severe CAD.

\section{P11}

PLOIDY ANALYSIS OF MENINGIOMAS AND ITS CORRELATION WITH NUCLEAR ATYPIA

Markidou S. (1), Karatzia S. (1), Georgoulakis I. (1), Maounis N. (2), Kazantzis D. (1), Paleologou A. (1), Damaskou V. (1), Delaportas G. (1), Mahera H. (3)

(1) Saint Savas Hospital, Athens, (2) Sismanoglion Hospital, Athens, (3) General District Hospital KAT, Kifissia, Greece

The aim of this study was the presentation of preliminary results on DNA ploidy analysis of intracranial meningiomas and its correlation with nuclear atypia of the tumor.

During the last six years 34 cases of intracranial meningiomas were studied. Fresh tumor imprints were prepared intraoperatively. Image analysis cytometry was performed on Feulgen stained imprints using the SAMBA image analysis system. DNA histograms were evaluated according the DNA Index and 5c-ER. Thirty cases were histologically characterized as meningotheliomatous, transitional or psammomatous meningiomas. Out of these cases, 27 with no cytologic atypia were diploid and the remaining 3 with nuclear pleomorphism were polyploid (one diploid and one tetraploid peak).

Four cases were histologically diagnosed as atypical meningiomas due to histologic and cytologic atypia or brain invasion. Nuclear pleomorphism including bizarre nuclei was evident. DNA analysis in three cases revealed polyploid histograms, i.e. one diploid and one tetraploid peak with negative 5c-ER. In one of these cases the recurrence tumor was available for DNA analysis. Again the DNA histogram showed one diploid and one tetraploid peak but positive $5 \mathrm{c}$-ER. The fourth case of atypical meningioma was aneuploid with one diploid peak, a second peak with DNA Index 1.7 and negative 5c-ER.

In summary, in our material most of the histologically benign meningiomas $(90 \%)$ were diploid and $10 \%$ polyploid. The atypical meningiomas were polyploid (3 cases) or aneuploid (one case). Nuclear pleomorphism seems to be mostly due to polyploidy, explaining why nuclear pleomorphism and hyperchromasia alone are of limited diagnostic value and considered by some authors to be of degenerative nature. 


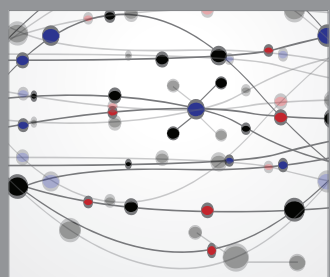

The Scientific World Journal
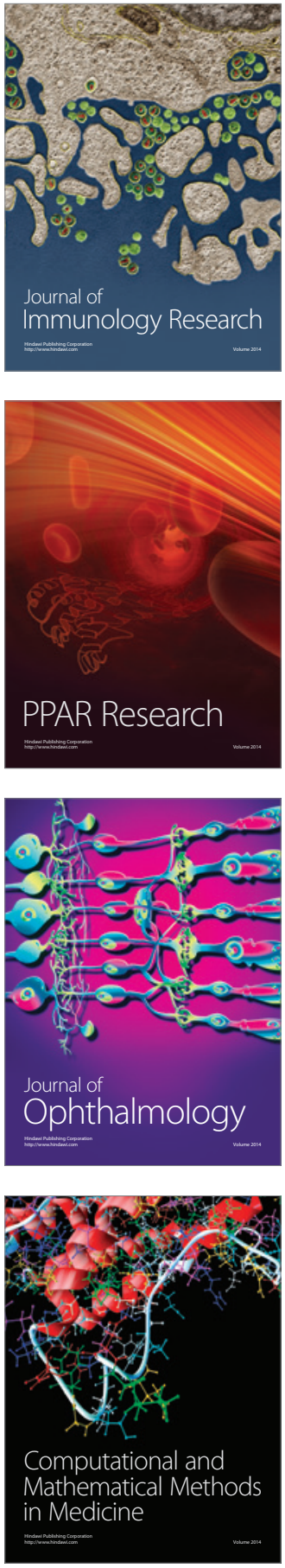

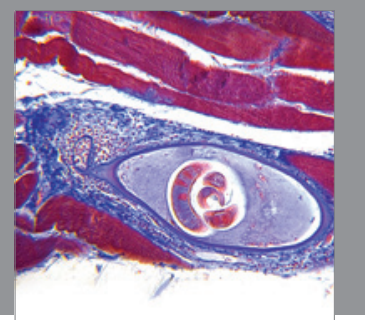

Gastroenterology

Research and Practice
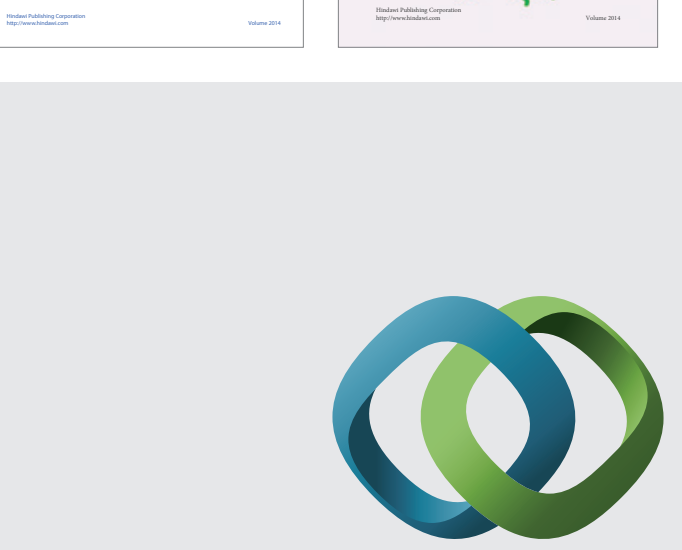

\section{Hindawi}

Submit your manuscripts at

http://www.hindawi.com
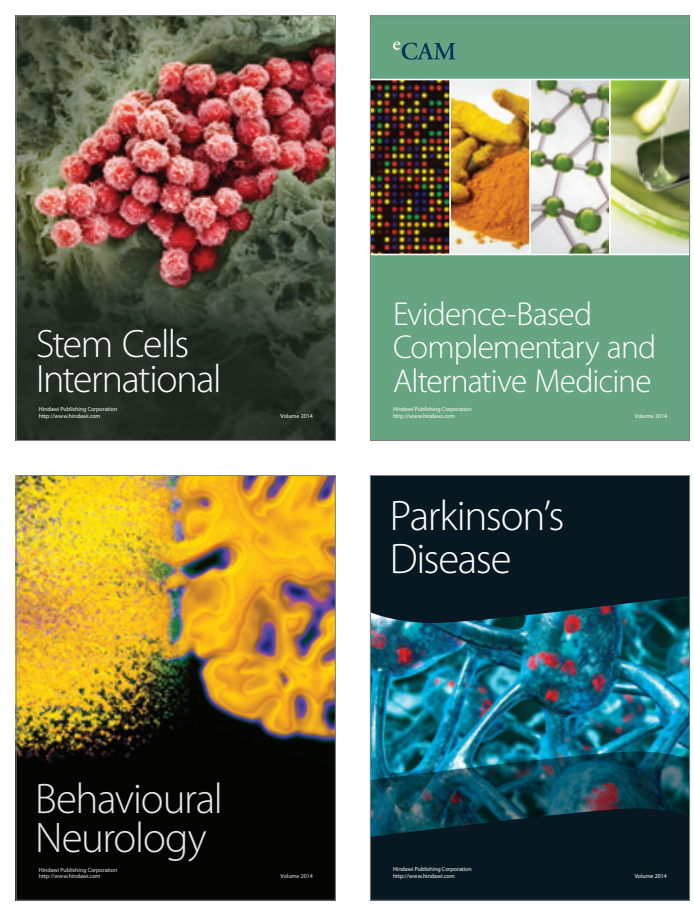

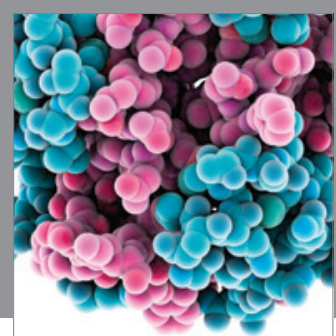

Journal of
Diabetes Research

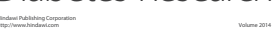

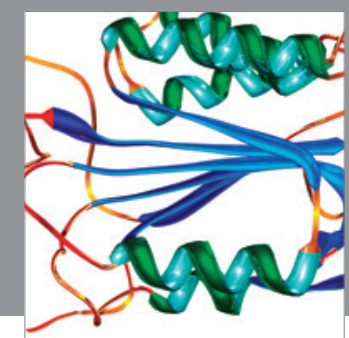

Disease Markers
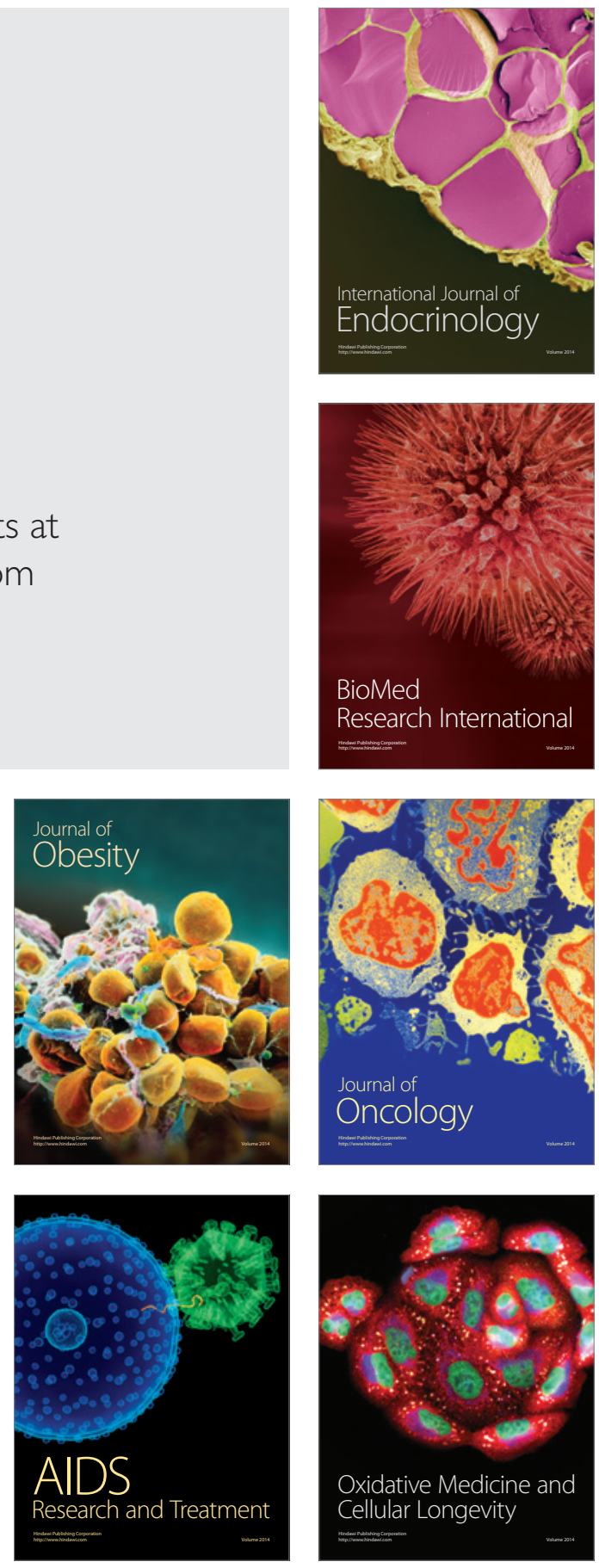